\author{
ANNALES \\ POLONICI MATHEMATICI \\ $96.3(2009)$
}

\title{
Quantifier elimination, valuation property and preparation theorem in quasianalytic geometry via transformation to normal crossings
}

\author{
by KRZYsztof Jan NowaK (Kraków)
}

\begin{abstract}
This paper investigates the geometry of the expansion $\mathcal{R}_{Q}$ of the real field $\mathbb{R}$ by restricted quasianalytic functions. The main purpose is to establish quantifier elimination, description of definable functions by terms, the valuation property and preparation theorem (in the sense of Parusiński-Lion-Rolin). To this end, we study nonstandard models $\mathcal{R}$ of the universal diagram $T$ of $\mathcal{R}_{Q}$ in the language $\mathcal{L}$ augmented by the names of rational powers. Our approach makes no appeal to the Weierstrass preparation theorem, upon which the majority of fundamental results in analytic geometry rely, but which is unavailable in the general quasianalytic geometry. The basic tools applied here are transformation to normal crossings and decomposition into special cubes. The latter method, developed in our earlier article [Ann. Polon. Math. 96 (2009), 65-74], combines modifications by blowing up with a suitable partitioning. Via an analysis of $\mathcal{L}$-terms and infinitesimals, we prove the valuation property for functions given by $\mathcal{L}$-terms, and next the exchange property for substructures of a given model $\mathcal{R}$. Our proofs are based on the concepts of analytically independent as well as active and non-active infinitesimals, introduced in this article. Further, quantifier elimination for $T$ is established through model-theoretic compactness. The universal theory $T$ is thus complete and o-minimal, and $\mathcal{R}_{Q}$ is its prime model. Under the circumstances, every definable function is piecewise given by $\mathcal{L}$-terms, and therefore the previous results concerning $\mathcal{L}$-terms generalize immediately to definable functions. In this fashion, we obtain the valuation property and preparation theorem for quasi-subanalytic functions. Finally, a quasi-subanalytic version of Puiseux's theorem with parameter is demonstrated.
\end{abstract}

1. Introduction. As in our previous paper [28], we begin - following Bierstone-Milman [4,3]-by fixing a family $\mathcal{Q}=\left(\mathcal{Q}_{m}\right)_{m \in \mathbb{N}}$ of sheaves of local $\mathbb{R}$-algebras of smooth functions on $\mathbb{R}^{m}$. For each open subset $U \subset \mathbb{R}^{m}$, $\mathcal{Q}(U)=\mathcal{Q}_{m}(U)$ is thus a subalgebra of the algebra $\mathcal{C}_{m}^{\infty}(U)$ of real smooth

2000 Mathematics Subject Classification: Primary 32S45, 14P15, 32B20; Secondary 03C10, 26E10, $03 \mathrm{C} 64$.

Key words and phrases: quasianalytic functions, special cubes, special modifications, analytically independent infinitesimals, active and non-active infinitesimals, valuation property, quantifier elimination, preparation theorem. 
functions on $U$. By a $Q$-function we mean any function $f \in \mathcal{Q}(U)$. Similarly,

$$
f=\left(f_{1}, \ldots, f_{k}\right): U \rightarrow \mathbb{R}^{k}
$$

is called a $Q$-mapping if so are its components $f_{1}, \ldots, f_{k}$. We impose the following six conditions on this family of sheaves:

1. each algebra $\mathcal{Q}(U)$ contains the restrictions of polynomials;

2. $\mathcal{Q}$ is closed under composition, i.e. the composition of Q-mappings is a Q-mapping (whenever it is well defined);

3. $\mathcal{Q}$ is closed under taking inverse, i.e. if $\varphi: U \rightarrow V$ is a Q-mapping between open subsets $U, V \subset \mathbb{R}^{m}, a \in U, b \in V$ and if $\frac{\partial \varphi}{\partial x}(a) \neq 0$, then there are neighbourhoods $U_{a}$ and $V_{b}$ of $a$ and $b$, respectively, and a Q-diffeomorphism $\psi: V_{b} \rightarrow U_{a}$ such that $\varphi \circ \psi$ is the identity mapping on $V_{b}$;

4. $\mathcal{Q}$ is closed under differentiation;

5. $\mathcal{Q}$ is closed under division by a coordinate, i.e. if $f \in \mathcal{Q}(U)$ and $f\left(x_{1}, \ldots, x_{i-1}, a_{i}, x_{i+1}, \ldots, x_{m}\right)=0$ as a function in the variables $x_{j}$, $j \neq i$, then $f(x)=\left(x_{i}-a_{i}\right) g(x)$ with some $g \in \mathcal{Q}(U)$;

6. $\mathcal{Q}$ is quasianalytic, i.e. if $f \in \mathcal{Q}(U)$ and the Taylor series $\widehat{f}_{a}$ of $f$ at a point $a \in U$ vanishes, then $f$ vanishes in the vicinity of $a$.

By means of Q-mappings, one can build, in the ordinary manner, the category Q of Q-manifolds and Q-mappings, which is a subcategory of that of smooth manifolds and smooth mappings. Similarly, Q-semianalytic and Q-subanalytic sets can be defined. Consider now the expansion $\mathcal{R}_{Q}$ of the real field $\mathbb{R}$ by restricted Q-functions, i.e. functions of the form

$$
\widetilde{f}(x)= \begin{cases}f(x) & \text { if } x \in[-1,1]^{m}, \\ 0 & \text { otherwise }\end{cases}
$$

where $f(x)$ is a Q-function in the vicinity of the compact cube $[-1,1]^{m}$. The structure $\mathcal{R}_{Q}$ is model-complete and o-minimal (cf. [36, 35, 28]). The definable subsets in $\mathcal{R}_{Q}$ coincide with those subsets in $\mathbb{R}^{m}$ that are Q-subanalytic in a semialgebraic compactification of $\mathbb{R}^{m}$.

In order to investigate $\mathcal{L}$-terms of the structure $\mathcal{R}_{Q}$, we shall consider the universal diagram $T$ of the structure $\mathcal{R}_{Q}$ in the language $\mathcal{L}$ of restricted quasianalytic functions augmented by the names of rational powers (i.e. the set of all universal $\mathcal{L}$-sentences that are true in $\mathcal{R}_{Q}$ ). We impose the ordinary postulates on the reciprocal function $1 / x$ and roots, namely

$$
\begin{aligned}
& x \cdot 1 / x=1 \quad \text { if } x \neq 0 \quad \text { and } \quad 1 / x=0 \quad \text { if } x=0, \\
& (\sqrt[n]{x})^{n}=x \quad \text { if } x \geq 0 \quad \text { and } \quad \sqrt[n]{x}=0 \quad \text { if } x<0 .
\end{aligned}
$$

The role of the function symbols attached to the language of restricted quasianalytic functions can be explained as follows. The reciprocal function $1 / x$ 
is indispensable when inverting transformation by blowing up, and roots are indispensable when inverting substitution of powers.

Our analysis of $\mathcal{L}$-terms and infinitesimals in non-standard models of $T$ is based on transformation to normal crossings and decomposition into special cubes. The latter method, introduced in our article [28] for relatively compact Q-semianalytic sets, combines modifications by blowing up along smooth centres with a suitable partitioning. It carries over, as shown in Section 2, to any sets described by $\mathcal{L}$-terms, both in the structure $\mathcal{R}_{Q}$ and in non-standard models of $T$. Generally, in our non-standard analysis, we are interested in finding suitable special modifications which take into account a tuple of infinitesimals. A counterpart of this problem in the classical subanalytic geometry is to find a finite (or locally finite) family of suitable modifications whose targets cover a space under consideration. The major part of Sections 2 and 3 will be concerned with such problems.

In Section 2, the notion of analytically independent infinitesimals is introduced. There we deal, inter alia, with the desingularization of $\mathcal{L}$-terms by special modifications and a modification of a Q-function to a regular one with respect to one distinguished variable. Sections 3 and 4 are devoted to the study of our concept of an active and a non-active infinitesimal, which is crucial for the whole work. An infinitesimal $\mu$ is active over a finite set $\lambda$ of infinitesimals if, for a certain $\mathcal{L}$-term $t(x, y)$ which is linear with respect to the variable $y$, the valuation of $t(\lambda, \mu)$ is not in the valuation group of the structure $\langle\lambda\rangle$ generated by the set $\lambda$.

In the third section, we treat the case of a non-active infinitesimal. We consider certain modifications, which are linear with respect to the distinguished variable that corresponds to a non-active infinitesimal. Most of the theorems from this section ensure that a $\mathrm{Q}$-function or an $\mathcal{L}$-term in question can be improved after applying such modifications; these are for instance: the theorem on behaviour of an $\mathcal{L}$-term at a non-active infinitesimal or the exchange property for a non-active infinitesimal. The latter amounts to solving, with respect to one distinguished variable, say $y$, an equation given by an $\mathcal{L}$-term. It is worth pointing out that we have reduced the problem of solving such an equation to that of solving a linear equation and to the implicit function theorem.

Let us mention that a linearization of an analytic equation with respect to one distinguished variable $y$ can be achieved in the classical analytic geometry by means of the Weierstrass preparation theorem and the Abhyankar-Jung theorem (cf. [1, 17, 33]). Whereas the former reduces a given equation, after blowing up the remainder variables $x$, to a polynomial one with respect to $y$, the latter allows one, after modifying the remainder variables $x$ by transformation to normal crossings and substitution of powers, to decompose the polynomial into a finite product of linear 
factors of the form $y-a_{i}(x)$ with some analytic functions $a_{i}(x)$ (see e.g. $[34,37,31,33])$.

We wish to emphasize the linear character of the definition of a non-active infinitesimal as well as of modifications with respect to a distinguished variable, on which our theory has been built. Not only does it enable us to avoid the Weierstrass preparation theorem, but also plays a vital role in the proof of the valuation property. Moreover, transformation to normal crossings, when taking into account the coordinate functions, propagates linearly through the valuation group. Note that classically, as initiated by Łojasiewicz [21] (see also $[7,8]$ ), the combination of the Weierstrass preparation and TarskiSeidenberg theorems was a basic tool in the geometry of semianalytic and subanalytic sets.

An active infinitesimal cannot be handled in a similar way to a non-active one. Section 4 is devoted to the study of an active infinitesimal. We prove, inter alia, the theorem on behaviour of an $\mathcal{L}$-term at a regular sequence of infinitesimals, the valuation property for $\mathcal{L}$-terms and, eventually, the exchange property for substructures of a given model $\mathcal{R}$ of the universal theory $T$. It means that the span operation on the family of all subsets of the model $\mathcal{R}$ is a pregeometry on $\mathcal{R}$. This enables us to introduce a general notion of independence for subsets of $\mathcal{R}$ as well as - by analogy with the dimension of vector spaces or with the transcendence degree of field extensions - the notion of rank and relative rank for substructures of $\mathcal{R}$. Following ZariskiSamuel [41, Chap. II, §12], we express the former as the notion of a free set, which turns out to coincide, for the case of a set of infinitesimals, with our notion of analytical independence.

What is crucial for our analysis of active infinitesimals is a delicate theorem on an active infinitesimal from Section 4. For its proof in the case of real analytic functions, we can make use of diagonal series. In the general quasianalytic settings, however, we are not able to apply this technique, because we do not know whether the diagonal series of a quasianalytic function determines a quasianalytic function too. Our further deduction is as follows: we derive from the theorem in question the valuation and exchange properties for $\mathcal{L}$-terms; next, in Section 5, quantifier elimination for the theory $T$ under study is established.

The line of reasoning mentioned above could be reversed. Quantifier elimination for a universal theory in the language $\mathcal{L}$, established by Rambaud [35], implies that our theory $T$ coincides with the o-minimal theory of the structure $\mathcal{R}_{Q}$. By a theorem of Herbrand [14] (see Section 6), every definable function is then piecewise given by $\mathcal{L}$-terms. Therefore the exchange property for $\mathcal{L}$-terms holds, and thus the theorem follows immediately.

One of the reasons why we provide a separate, direct proof of this theorem is that - in our opinion-Rambaud's paper [35] contains an essential 
gap. His proof of quantifier elimination from Section 8 is based on Corollary 6.14 which, in turn, relies on Proposition 6.11 about stable families of infinitesimals (to the effect that a stable family $x_{1}, \ldots, x_{n}$ of infinitesimals remains stable after attaching an infinitesimal $y$ independent of $x_{1}, \ldots, x_{n}$ ). But the application of Lemma 5.7 in the proof of the proposition is valid only if the conclusion of the proposition holds. This brings about a vicious circle, and we do not know how to repair it.

We are able to provide a complete proof of the theorem for the particular quasianalytic case where the valuations of the infinitesimals $\lambda_{1}, \ldots, \lambda_{m}$ are linearly independent over $\mathbb{Q}$. Let us mention that our proof for this case is much more complicated. Instead of diagonal series, we use a recursive method of admissible annihilation of a sufficient yet finite number of diagonal Taylor coefficients of the function under study.

In a previous version of this article (see also [29]), we posed the following problem which generalizes the one about diagonal series:

Let $f$ be a $Q$-function at $0 \in \mathbb{R}^{k}$ with Taylor series $\hat{f}$. Split the set $\mathbb{N}^{k}$ of exponents into two disjoint subsets $A$ and $B, \mathbb{N}^{k}=A \cup B$, and decompose the formal series $\hat{f}$ into the sum of two formal series $G$ and $H$, supported by $A$ and $B$, respectively. Do there exist two $Q$-functions $g$ and $h$ at $0 \in \mathbb{R}^{k}$ with Taylor series $G$ and $H$, respectively?

and related it to the question whether polynomials are dense in a certain Hilbert space associated with a quasianalytic Denjoy-Carleman class, investigated by Thilliez [38] in connection with his proof of Carleman's theorem on the failure of surjectivity for the Borel mapping. A slight modification of his proof provides a one-dimensional counter-example (given by a lacunary Taylor series) to the foregoing problem. We know as yet no counter-examples for the case of diagonal splitting of the set of Taylor coefficients. In case the answer to this problem turns out to be an affirmative, our proof of the analytic case of the theorem would extend verbatim to the general quasianalytic settings.

The Hilbert space considered by Thilliez (an analogue of Sobolev spaces of infinite order of type $l_{2}$ ) allowed him to handle simultaneously an infinite number of derivatives. Earlier, O'Farrell [30] had studied in general the density of polynomials in Banach spaces of ultradifferentiable functions of type $l_{p}, 1 \leq p \leq \infty$, embracing that of Thilliez. He had given the affirmative answer for functions of one variable using a principle of dominated convergence and the Poisson integral. Nevertheless, it seems to remain valid for the case of several variables as well. Let us mention that certain Banach algebras of this type, with particular reference to the non-analytic quasianalytic case, were also investigated by Dales-Davie [6]. 
In Section 5, we apply the foregoing results along with model-theoretic compactness to the problem of inversion of general special modifications. It will turn out that the requirement for the inverse mapping $\psi$ of a special modification $\varphi$ we impose in Section 2 is no constraint on special cubes at all, because it is fulfilled by every special modification. We need this inversion theorem and Gabrielov's closure theorem in order to establish quantifier elimination for the theory $T$. In fact, we shall prove that if a set $E$ is described by $\mathcal{L}$-terms, so is its projection. Our proof makes use of model-theoretic compactness again. Consequently, the theory $T$ is complete and o-minimal, and the standard model $\mathcal{R}_{Q}$ is its prime model.

Quantifier elimination and an elementary universal axiomatization for the expansion of the real field by restricted analytic functions were established by van den Dries-Macintyre-Marker in the language augmented by the names of the reciprocal function $1 / x$ and the roots (see [10], and also $[9,24,25])$. Recently, Rambaud [35] proved a theorem of this kind for the quasianalytic setting, which was also a stimulus for our writing this article. In particular, the theorem on lowering order from Section 3 was inspired by the relevant idea from his Lemma 5.8. Rambaud investigates families of so-called stable infinitesimals, which play a key role in his proof. He makes use of certain desingularization algorithms and an embedding of a model under consideration into an ultrapower of the real field.

The fact that a universal theory $T$ admits quantifier elimination has weighty model-theoretic and geometric consequences, implying in particular that every definable function is piecewise given by terms (a theorem of Herbrand [14]). Therefore all the results we have previously proved for $\mathcal{L}$-terms remain valid for definable functions. Section 6 provides a brief exposition of several applications. First, the valuation property for definable functions is stated. Hence, through model-theoretic compactness and definable choice (which is available for o-minimal structures), one can derive the preparation theorem in the sense of Parusiński-Lion-Rolin (see [12, 26]). Finally, we demonstrate a subanalytic version of Puiseux's theorem with parameter as well as its immediate consequence, piecewise uniform asymptotics.

We conclude this section with some useful remarks.

REMARKS 1.1. (1) Let $\Phi$ be an arbitrary semialgebraic diffeomorphism of $\mathbb{R}^{m}$ onto $(-1,1)^{m}$. In view of Gabrielov's complement theorem, $E \subset \mathbb{R}^{m}$ is a definable subset of the structure $\mathcal{R}_{Q}$ iff $\Phi(E)$ is a (bounded) Q-subanalytic subset in $\mathbb{R}^{m}$.

(2) Condition 4 imposed on the family of quasianalytic functions is a direct consequence of condition 5 . We must show that if $\widetilde{f}(x)$ is a restricted Q-function, so is each partial derivative $\frac{\partial f}{\partial x_{i}}(x)$. We check it for $i=m$. Since the function $f(x)$ is Q-analytic in the vicinity of $[-1,1]^{m}$, the function 


$$
g(x, y):=f\left(x_{1}, \ldots, x_{m-1}, x_{m}+y\right)-f(x)
$$

is Q-analytic in the vicinity of $[-1,1]^{m} \times[-\delta, \delta] \subset \mathbb{R}^{m+1}$ with some $\delta>0$. It follows from condition 5 that $g(x, y)=y h(x, y)$ for a function $h(x, y)$ Q-analytic in the vicinity of $[-1,1]^{m} \times[-\delta, \delta]$. Hence

$$
\frac{\partial f}{\partial x_{m}}(x)=\frac{\partial g}{\partial y}(x, 0)=h(x, 0)
$$

for $x$ in the vicinity of $[-1,1]^{m}$, which is the desired result.

(3) Under condition 6 of quasi-analyticity, condition 5 for convex subsets $U$ is equivalent to the following one: if the Taylor series of $f \in \mathcal{Q}(U)$ at a point $a \in U$ is divisible by $x_{i}-a_{i}$, then $f(x)=\left(x_{i}-a_{i}\right) g(x)$ with some $g \in \mathcal{Q}(U)$.

(4) Although it is well-known that every model $\mathcal{R}$ of the theory $T$ is a real closed field (see e.g. [23]), we shall not use this fact in our approach.

(5) The interpretation $f^{\mathcal{R}}$ of each restricted Q-function $\tilde{f}$ in any model $\mathcal{R}$ of the theory $T$ is an infinitely differentiable function, and we have

$$
\frac{\partial f^{\mathcal{R}}}{\partial x_{i}}=\left(\frac{\partial f}{\partial x_{i}}\right)^{\mathcal{R}}
$$

Indeed, if $f(x)$ is Q-analytic in the vicinity of $[-1,1]^{m}$, then (as in Remark 2)

$$
f\left(x_{1}, \ldots, x_{m-1}, x_{m}+y\right)-f(x)=y h(x, y)=y[h(x, 0)+y k(x, y)]
$$

for certain functions $h(x, y)$ and $k(x, y)$ which are Q-analytic in the vicinity of $[-1,1]^{m} \times[-\delta, \delta]$ with some $\delta>0$. Hence

$$
\frac{\partial f^{\mathcal{R}}}{\partial x_{m}}(x)=h^{\mathcal{R}}(x, 0)=\left(\frac{\partial f}{\partial x_{i}}\right)^{\mathcal{R}}(x) .
$$

(6) Similarly, making use of Taylor's formula, we have

$$
f\left(x_{1}, \ldots, x_{m-1}, x_{m}+y\right)-\sum_{j=0}^{n} \frac{1}{j !} \cdot \frac{\partial^{j} f}{\partial x_{m}^{j}}(x) \cdot y^{j}=y^{n+1} \cdot h(x, y)
$$

for a function $h(x, y)$ Q-analytic in the vicinity of $[-1,1]^{m} \times[-\delta, \delta]$ with some $\delta>0$.

2. Special cubes and analytically independent infinitesimals. We proved in [28] the following

TheOREM 2.1 (on decomposition into special cubes). Every bounded Qsemianalytic subset $E$ in $\mathbb{R}^{m}$ is a finite union of special cubes $S_{j}$, i.e. subsets in $\mathbb{R}^{m}$ of the form

$$
S_{j}=\varphi_{j}\left((-1,1)^{d_{j}}\right)
$$

where $\varphi_{j}(x)$ is a Q-mapping from the vicinity of $[-1,1]^{d_{j}}$ into $\mathbb{R}^{m}$ such that the restriction of $\varphi_{j}$ to $(-1,1)^{d_{j}}$ is a diffeomorphism onto $S_{j}$. 
Furthermore, each of those special cubes $S_{j}$ and the inverse mappings

$$
\psi_{j}: S_{j} \rightarrow(-1,1)^{d_{j}}
$$

to the associated Q-diffeomorphisms $\varphi_{j}$ are piecewise given by terms in the language of restricted Q-analytic functions augmented by the name of the reciprocal function $1 / x$.

REMARK 2.2. The inverse mappings $\psi_{j}$ to the diffeomorphisms $\varphi_{j}$ are piecewise given by terms in the language of restricted Q-functions augmented by the name of the reciprocal function $1 / x$, because - roughly speakingthe mappings $\psi_{j}$ have been locally built in the process of blowing up as a successive superposition of restricted Q-functions and of the reciprocal function $1 / x$ off the zero argument.

Corollary 2.3. Let $F \subset \mathbb{R}^{m}$ be a bounded subset described by $\mathcal{L}$-terms and $t(x)=t\left(x_{1}, \ldots, x_{m}\right)$ be an $\mathcal{L}$-term. Then the part of the graph of $t(x)$ lying over $F$ is a finite union of special cubes $S_{i}$ in $\mathbb{R}^{m} \times \mathbb{R}$ of the form

$$
S_{i}=\varphi_{i}\left((-1,1)^{d_{i}}\right),
$$

where $\varphi_{i}(x)$ is a $Q$-mapping from the vicinity of $[-1,1]^{d_{i}}$ into $\mathbb{R}^{m} \times \mathbb{R}$ such that the restriction of $\varphi_{i}$ to $(-1,1)^{d_{i}}$ is a $Q$-diffeomorphism onto $S_{i}$.

Furthermore, each of those special cubes $S_{i}$ and the inverse mappings $\psi_{i}: S_{i} \rightarrow(-1,1)^{d_{i}}$ to $\varphi_{i}$ are piecewise given by $\mathcal{L}$-terms.

Remark 2.4. Decomposition into special cubes yields the above corollary according to the following observation. After adding new variables (one for each occurrence of a function symbol involved in a given $\mathcal{L}$-term), the graph of this term and the sets described by a finite number of $\mathcal{L}$-terms are bijective projections of certain Q-semianalytic subsets, and the inverse mappings to those projections are given by $\mathcal{L}$-terms. Note that if a subset contained in the domain of such a projection is described by $\mathcal{L}$-terms, so is its image under this projection. In this fashion, techniques related to Q-semianalytic sets can be adapted to the sets described by $\mathcal{L}$-terms.

Remark 2.5. Given an $\mathcal{L}$-term $t(x)=t\left(x_{1}, \ldots, x_{m}\right)$, there exists a partition of $\mathbb{R}^{m}$ into finitely many Q-submanifolds described by $\mathcal{L}$-terms, such that the restriction of the function given by $t(x)$ to each of these Qsubmanifolds is smooth (i.e. $C^{\infty}$ ).

Unless otherwise stated we shall deal with special cubes $(S, \varphi)$ such that

- $\varphi$ is a Q-mapping in the vicinity of $[-1,1]^{d}$ (or sometimes $[0,1]^{d}$ ) which is a diffeomorphism of $(-1,1)^{d}$ onto $S$;

- the inverse mapping $\psi$ to this diffeomorphism is piecewise given by $\mathcal{L}$-terms. 
It will turn out that the above requirement for the inverse mapping $\psi$ is no constraint on special cubes at all, because it is fulfilled by every special cube (Proposition 5.4). We shall also consider special cubes described above, but which are the diffeomorphic images of arbitrary cubes in $\mathbb{R}^{d}$, and especially of the cubes $(0,1)^{d}$.

We may regard a special cube $\varphi:(0,1)^{d} \rightarrow S$ as a kind of modification of the set $S$. When we look at the special cube $(S, \varphi)$ in this manner, we shall call $\varphi$ a special modification.

We now fix a model $\mathcal{R}$ of the universal theory $T$ in the language $\mathcal{L}$. Every $\mathcal{L}$-substructure of $\mathcal{R}$ is a model of $T$. We always regard the standard model $\mathcal{R}_{Q}$ as a substructure of $\mathcal{R}$. Since the decompositions into special cubes we deal with are described by $\mathcal{L}$-terms (both a special modification $\varphi$ and its inverse $\psi$ ), they are preserved by passage to any model $\mathcal{R}$ of $T$ :

$$
E^{\mathcal{R}}=\bigcup_{j} S_{j}^{\mathcal{R}} \quad \text { and } \quad(\operatorname{graph} t(x) \cap(F \times \mathbb{R}))^{\mathcal{R}}=\bigcup_{j} S_{j}^{\mathcal{R}} .
$$

For simplicity of notation, we shall usually omit the superscript ${ }^{\mathcal{R}}$ referring to the interpretations in a model $\mathcal{R}$, which will not lead to confusion.

We now turn to an analysis of infinitesimals of the model $\mathcal{R}$. We say that infinitesimals $\lambda=\left(\lambda_{1}, \ldots, \lambda_{m}\right) \in \mathcal{R}$ are analytically dependent if $\lambda$ lies in a special cube $S=\varphi\left((0,1)^{d}\right)$ with $d<m$. We call infinitesimals $\lambda$ analytically independent if they are not analytically dependent. Analytical independence is preserved, of course, under permutation of infinitesimals. We say that a subset $A$ in $\mathcal{R}$ is analytically independent if every finite subset $A$ in $\mathcal{R}$ consists of analytically independent infinitesimals. If $A \subset B$ and the set $B$ is analytically independent, so is $A$.

For any subset $A \subset \mathcal{R},\langle A\rangle$ denotes the substructure of $\mathcal{R}$ generated by $A$. Every finitely generated model of $T$ has, of course, a finite, analytically independent set of generators.

The convex hull of $\mathbb{R}$ in $\mathcal{R}$ is a valuation ring $V$ of bounded (with respect to $\mathbb{R}$ ) elements in $\mathcal{R}$; its maximal ideal $\mathfrak{m}$ consists of all infinitesimals in $\mathcal{R}$. The valuation $v$ induced by $V$ is called the standard valuation on the field $\mathcal{R}$; its value group $\Gamma_{\mathcal{R}}$ is a $\mathbb{Q}$-vector space. In order to investigate the valuation $v$, we shall need several results about Q-functions, stated and proved in this and the next section.

Now we state yet another corollary to the theorem on decomposition into special cubes, which is a direct consequence of Corollary 2.3, applied to the graph of a given term $t(x)$.

Corollary 2.6 (Desingularization of an $\mathcal{L}$-term). Consider an $\mathcal{L}$-term $t(x)$ and positive analytically independent infinitesimals $\lambda=\left(\lambda_{1}, \ldots, \lambda_{m}\right)$. If $t(\lambda)$ is bounded, then there exist a special cube $S=\varphi\left((0,1)^{m}\right) \subset \mathbb{R}^{m}, a$ 
Q-function $f\left(x^{\prime}\right)$ in the vicinity of $[0,1]^{m}$, and positive infinitesimals $\lambda^{\prime}=$ $\left(\lambda_{1}^{\prime}, \ldots, \lambda_{m}^{\prime}\right)$ such that $\lambda=\varphi\left(\lambda^{\prime}\right) \in S$ and

$$
t^{\varphi}\left(x^{\prime}\right):=t\left(\varphi\left(x^{\prime}\right)\right)=f\left(x^{\prime}\right) \quad \text { for all } x^{\prime} \in(0,1)^{m} .
$$

We can rephrase the conclusion of Corollary 2.6 as follows.

One can find a special modification

$$
\varphi:(0,1)^{m} \rightarrow \mathbb{R}^{m} \quad \text { with } \quad \lambda=\varphi\left(\lambda^{\prime}\right) \text { for some } \lambda^{\prime} \in(0,1)^{m}
$$

such that the superposition $f:=t \circ \varphi$ extends to a $Q$-function in the vicinity of $[0,1]^{m}$; in particular, $t(\lambda)=f\left(\lambda^{\prime}\right)$.

The next theorem will be crucial for investigation of $y$-regular Q-functions. Its proof makes use of the noetherianity of the rings of formal power series.

Proposition 2.7. Let $f_{n}:[-1,1]^{m} \rightarrow \mathbb{R}, n \in \mathbb{N}$, be restricted Qfunctions, not all of which vanish identically, and let $\lambda=\left(\lambda_{1}, \ldots, \lambda_{m}\right)$ be positive analytically independent infinitesimals. Then one can find a special cube

$$
S=\varphi\left((0,1)^{m}\right) \subset \mathbb{R}^{m}
$$

with special modification $\varphi(x)$ Q-analytic in the vicinity of the cube $[0,1]^{m}$ and a composite of successive blowings-up, and positive infinitesimals $\lambda^{\prime}=$ $\left(\lambda_{1}^{\prime}, \ldots, \lambda_{m}^{\prime}\right)$ such that $\lambda=\varphi\left(\lambda^{\prime}\right) \in S$ and

$$
f_{n}^{\varphi}\left(x^{\prime}\right):=\left(f_{n} \circ \varphi\right)\left(x^{\prime}\right)=x^{\prime \alpha} u_{n}\left(x^{\prime}\right), \quad n \in \mathbb{N},
$$

in the vicinity of $[0,1]^{m}$, where $\alpha \in \mathbb{N}^{m}$, the functions $u_{n}\left(x^{\prime}\right)$ are $Q$-analytic in the vicinity of $[0,1]^{m}$ and $u_{k}(0) \neq 0$ for a certain $k \in \mathbb{N}$.

REMARK 2.8. Note that the inverse mapping $\psi: S \rightarrow(0,1)^{m}$ to the special modification $\varphi$ is piecewise given by terms in the language of restricted Q-functions augmented by the name of the reciprocal function $1 / x$. Such a special modification $\varphi$ can be achieved by transformation to normal crossings by blowing up combined with a suitable partitioning on each successive stage of the process of blowing up (according to our method of decomposition into special cubes, presented in [28]).

For the proof of Proposition 2.7, consider the ideal $\mathcal{I} \subset \mathbb{R}[[x]]$ generated by the Taylor series at zero $\widehat{f}_{n}(x)$ of the functions $f_{n}(x)$ and take generators $\widehat{f}_{1}, \ldots, \widehat{f}_{N}$ of $\mathcal{I}$. One can simultaneously transform by blowing up the functions

$$
f_{1}(x), \ldots, f_{N}(x), x_{1}, \ldots, x_{m}
$$

to normal crossings, so that

$$
f_{n}^{\varphi}\left(x^{\prime}\right)=x^{\prime \beta_{n}} v_{n}\left(x^{\prime}\right), \quad v_{n}(0) \neq 0 \quad \text { for } n=1, \ldots, N,
$$


and the exponents $\beta_{1}, \ldots, \beta_{N}$ are totally ordered with respect to the partial ordering induced from $\mathbb{N}^{m}$ (i.e. $\alpha \leq \beta$ means $\alpha_{i} \leq \beta_{i}$ for all $i=1, \ldots, m$ )see e.g. [2, 4]. Putting

$$
\alpha:=\beta_{k}=\min \left\{\beta_{1}, \ldots, \beta_{N}\right\},
$$

we see that all the Taylor series at zero $\widehat{f_{n}^{\varphi}}\left(x^{\prime}\right)$ are divisible by $x^{\prime \alpha}$, whence so are the functions $f_{n}^{\varphi}\left(x^{\prime}\right)$ (by condition 5 imposed on our family of Qfunctions, which asserts that the quasianalytic family of functions is closed under division by a coordinate; see also Remark 1.1(3)). The conclusion can thus be achieved by our method of decomposition into special cubes [28], when one takes into account the following two observations:

- when transforming to normal crossings by blowing up, the successive inverse images of the infinitesimals $\lambda$ lie on no centre of the successive blowings-up, because they continue to be analytically independent;

- the final inverse image under the transformation of each orthant is a union of orthants, so that one may assume that the inverse image $\lambda^{\prime}$ of $\lambda$ lies in the first orthant.

By the $y$-order ord $f(x, y)$ of a Q-function $f(x, y)$ at zero we mean the smallest non-negative integer $n \in \mathbb{N}$ for which $\frac{\partial^{n} f}{\partial y^{r}}(0,0) \neq 0$, if such integers exist, or $\infty$ otherwise. We say that the function $f(x, y)$ is $y$-regular at zero if ord $f(x, y)<\infty$, i.e. $f(0, y) \not \equiv 0$. A useful fact, which is an immediate consequence of postulate 5 imposed on the family of Q-functions, is stated below.

LEMMA 2.9. If $f(x, y):[-1,1]^{m} \times[-1,1] \rightarrow \mathbb{R}$ is a restricted $Q$-function such that each partial derivative

$$
\frac{\partial^{n} f}{\partial y^{n}}(x, 0) \quad \text { for } n=0,1,2, \ldots
$$

is divisible by $x^{\alpha}$, then so is the function $f(x, y)$.

Hence and by the foregoing proposition applied to the sequence of Qfunctions $f_{n}(x):=\frac{\partial^{n} f}{\partial y^{n}}(x, 0)$ for $n=0,1,2, \ldots$, we obtain

Corollary 2.10 (Modification of a Q-function to a $y$-regular one). Let $f(x, y):[-1,1]^{m} \times[-1,1] \rightarrow \mathbb{R}$ be a restricted $Q$-function, $f \not \equiv 0$, and $\lambda=\left(\lambda_{1}, \ldots, \lambda_{m}\right)$ be positive analytically independent infinitesimals. Then one can find a special cube $S=\varphi\left((0,1)^{m}\right) \subset \mathbb{R}^{m}$ with $\varphi$ being a composite of successive blowings-up, and positive infinitesimals $\lambda^{\prime}=\left(\lambda_{1}^{\prime}, \ldots, \lambda_{m}^{\prime}\right)$ such that $\lambda=\varphi\left(\lambda^{\prime}\right) \in S$ and

$$
f^{\varphi}\left(x^{\prime}, y\right):=f\left(\varphi\left(x^{\prime}\right), y\right)=x^{\alpha} g\left(x^{\prime}, y\right)
$$

in the vicinity of $[0,1]^{m+1}$, where $\alpha \in \mathbb{N}^{m}$ and $g\left(x^{\prime}, y\right)$ is a $Q$-function $y$ regular at $(0,0) \in \mathbb{R}^{m} \times \mathbb{R}$. 
Corollary 2.11. Consider infinitesimals $\lambda=\left(\lambda_{1}, \ldots, \lambda_{m}\right)$ and $\mu$. If $v(\mu) \notin \Gamma_{\langle\lambda\rangle}$, then for any Q-function $f(x, y)$ at $(0,0) \in \mathbb{R}_{x}^{m} \times \mathbb{R}_{y}$ we have

$$
v(f(\lambda, \mu)) \in \Gamma_{\langle\lambda\rangle} \oplus \mathbb{N} \cdot v(\mu) .
$$

Indeed, we may assume that the infinitesimals $\lambda$ are analytically independent. In view of Corollary 2.10, we can assume that the function $f(x, y)$ is $y$-regular at zero, say, of $y$-order $n$. Then, in the vicinity of zero, we have

$$
f(x, y)=f_{0}(x)+f_{1}(x) y+\cdots+f_{n-1}(x) y^{n-1}+f_{n}(x, y) y^{n},
$$

where $f_{0}(x), \ldots, f_{n-1}(x), f_{n}(x, y)$ are Q-functions at zero and $f_{n}(0,0) \neq 0$. By the assumption of the corollary, the values

$$
v\left(f_{0}(\lambda)\right), v\left(f_{1}(\lambda) \mu\right), \ldots, v\left(f_{n-1}(\lambda) \mu^{n-1}\right), v\left(f_{n}(\lambda, \mu) \mu^{n}\right)=v\left(\mu^{n}\right)
$$

are pairwise distinct, and thus the assertion follows.

3. Active and non-active infinitesimals. We say that an infinitesimal $\mu$ is non-active over infinitesimals $\lambda=\left(\lambda_{1}, \ldots, \lambda_{m}\right)$ if for each $\mathcal{L}$-term $t(x)$ we have

$$
v(\mu-t(\lambda)) \in \Gamma_{\langle\lambda\rangle} .
$$

Otherwise, the infinitesimal $\mu$ is called active over $\lambda$. It is clear that if $\mu$ is non-active over $\lambda$, so is the infinitesimal $\mu^{\prime}=s(\lambda) \mu+t(\lambda)$ that is the value at $(\lambda, \mu)$ of any $y$-linear $\mathcal{L}$-term.

THEOREM 3.1 (on lowering $y$-order). Let $f(x, y)$ be a Q-function, $y$ regular at $(0,0) \in \mathbb{R}_{x}^{m} \times \mathbb{R}_{y}$ of y-order $n>0, \lambda=\left(\lambda_{1}, \ldots, \lambda_{m}\right)$ be positive infinitesimals and $\mu$ a positive infinitesimal, non-active over $\lambda$. Then there exist a special cube $S=\varphi\left((0,1)^{d}\right) \subset \mathbb{R}^{m}$ with $d \leq m$, a Q-function $\omega\left(x^{\prime}, y^{\prime}\right)$ in the vicinity of $[0,1]^{d} \times \mathbb{R}$, linear with respect to the last variable and with $\omega(0,0)=0$, a Q-function $g\left(x^{\prime}, y^{\prime}\right)$ in the vicinity of $[0,1]^{m+1}$ of $y^{\prime}$-order $<n$, positive infinitesimals $\lambda^{\prime}=\left(\lambda_{1}^{\prime}, \ldots, \lambda_{d}^{\prime}\right)$, a positive infinitesimal $\mu^{\prime}$, non-active over $\lambda^{\prime}$, and $\alpha \in \mathbb{N}^{m}$ such that $\lambda=\varphi\left(\lambda^{\prime}\right) \in S, \mu=\omega\left(\lambda^{\prime}, \mu^{\prime}\right)$ and

$$
f^{\sigma}\left(x^{\prime}, y^{\prime}\right):=f\left(\varphi\left(x^{\prime}\right), \omega\left(x^{\prime}, y^{\prime}\right)\right)=x^{\prime \alpha} g\left(x^{\prime}, y^{\prime}\right)
$$

in the vicinity of $[0,1]^{d+1}$; here $\sigma\left(x^{\prime}, y^{\prime}\right):=\left(\varphi\left(x^{\prime}\right), \omega\left(x^{\prime}, y^{\prime}\right)\right)$.

We may, of course, assume that the infinitesimals $\lambda$ are analytically independent, and in this case we have $d=m$. Our proof starts with the observation that the implicit function theorem yields a Q-function $\chi(x)$ at $0 \in \mathbb{R}^{m}$ such that

$$
\frac{\partial^{n-1} f}{\partial y^{n-1}}(x, \chi(x))=0 \quad \text { and } \quad \chi(0)=0 .
$$

After the $y$-linear change of variables $y^{\prime}=y-\chi(x)$, the infinitesimal $\mu^{\prime}:=$ 
$\mu-\chi(\lambda)$ remains non-active over $\lambda$. Therefore, one may assume that

$$
\frac{\partial^{n-1} f}{\partial y^{n-1}}(x, 0) \equiv 0 .
$$

Since $\mu$ is non-active over $\lambda, v(\mu)=v(t(\lambda))$ for some $\mathcal{L}$-term $t(x)$. Due to the desingularization of $\mathcal{L}$-terms (Corollary 2.6), there exist a special cube $S=\varphi\left((0,1)^{m}\right) \subset \mathbb{R}^{m}$ as described in the theorem, a Q-function $\xi(x)$ in the vicinity of $[0,1]^{m}$ and positive infinitesimals $\lambda^{\prime}=\left(\lambda_{1}^{\prime}, \ldots, \lambda_{m}^{\prime}\right)$ such that

$$
\lambda=\varphi\left(\lambda^{\prime}\right) \text { and } t(\lambda)=\xi\left(\lambda^{\prime}\right) .
$$

Through transformation to normal crossings by blowing up, one can also assume that $\xi(x)$ is a normal crossing at zero, whence

$$
v(\mu)=v(t(\lambda))=v\left(\left(\lambda^{\prime}\right)^{\alpha}\right)
$$

for some multi-index $\alpha \in \mathbb{N}^{m}$. We are thus reduced to the case where $v(\mu)=$ $v\left(\lambda^{\alpha}\right)$; then $\mu=(c+\epsilon) \lambda^{\alpha}$ with some $c \in \mathbb{R}$ and an infinitesimal $\epsilon$.

Consider now the $y$-linear change of variables $y^{\prime}=y-c x^{\alpha}$ and put

$$
\mu^{\prime}:=\mu-c \lambda^{\alpha}=\epsilon \lambda^{\alpha} ;
$$

obviously, $v\left(\mu^{\prime}\right)>v(\mu)$. Then

$$
f(x, y)=f\left(x, y^{\prime}+c x^{\alpha}\right)=: g\left(x, y^{\prime}\right)
$$

and

$$
\frac{\partial^{n-1} g}{\partial\left(y^{\prime}\right)^{n-1}}\left(x, y^{\prime}\right)=\frac{\partial^{n-1} f}{\partial y^{n-1}}\left(x, y^{\prime}+c x^{\alpha}\right) .
$$

Note that

$$
\frac{\partial^{n-1} g}{\partial\left(y^{\prime}\right)^{n-1}}(x, 0)=\frac{\partial^{n-1} f}{\partial y^{n-1}}\left(x, c x^{\alpha}\right) \not \equiv 0,
$$

because, by the initial reduction, $y=0$ is a unique solution near zero of the equation $\frac{\partial^{n-1} f}{\partial y^{n-1}}(x, y)=0$. Again, through transformation to normal crossings by blowing up, one can assume that the function $\frac{\partial^{n-1} g}{\partial\left(y^{\prime}\right)^{n-1}}(x, 0)$ is a normal crossing at $0 \in \mathbb{R}^{m}$, i.e.

$$
\frac{\partial^{n-1} g}{\partial\left(y^{\prime}\right)^{n-1}}(x, 0)=u(x) x^{\beta}
$$

for some $\beta \in \mathbb{N}^{m}$ and a Q-function $u(x)$ at $0 \in \mathbb{R}^{m}$ with $u(0) \neq 0$.

We now show that $\mu^{\prime \prime}:=\mu^{\prime} / \lambda^{\beta}$ is an infinitesimal too. Indeed, we have

$$
v\left(\lambda^{\beta}\right)=v\left(\frac{\partial^{n-1} g}{\partial\left(y^{\prime}\right)^{n-1}}(\lambda, 0)\right)=v\left(\frac{\partial^{n-1} f}{\partial y^{n-1}}\left(\lambda, c \lambda^{\alpha}\right)\right) .
$$

Since $\frac{\partial^{n} f}{\partial y^{n}}(0,0) \neq 0$, we get

$$
\frac{\partial^{n-1} f}{\partial y^{n-1}}(\lambda, \mu)=\frac{\partial^{n-1} f}{\partial y^{n-1}}(\lambda, 0)+\text { unit } \cdot \mu=\text { unit } \cdot \mu
$$


and

$$
\frac{\partial^{n-1} f}{\partial y^{n-1}}(\lambda, \mu)=\frac{\partial^{n-1} f}{\partial y^{n-1}}\left(\lambda, c \lambda^{\alpha}\right)+\text { unit } \cdot \mu^{\prime}
$$

Hence

$$
\frac{\partial^{n-1} f}{\partial y^{n-1}}\left(\lambda, c \lambda^{\alpha}\right)=\text { unit } \cdot \mu
$$

and thus $v\left(\lambda^{\beta}\right)=v(\mu)<v\left(\mu^{\prime}\right)$, as desired.

The above allows one to introduce yet another $y$-linear change of variables, namely $y^{\prime \prime}=y^{\prime} / x^{\beta}$. Then

$$
f(x, y)=g\left(x, y^{\prime}\right)=g\left(x, y^{\prime \prime} \cdot x^{\beta}\right)=: h\left(x, y^{\prime \prime}\right)
$$

whence we get

$$
\frac{\partial^{k} h}{\partial\left(y^{\prime \prime}\right)^{k}}\left(x, y^{\prime \prime}\right)=x^{k \cdot \beta} \cdot \frac{\partial^{k} g}{\partial\left(y^{\prime}\right)^{k}}\left(x, y^{\prime \prime} \cdot x^{\beta}\right) \quad \text { for all } k \in \mathbb{N} .
$$

We have, in particular, the equalities

$$
\frac{\partial^{k} h}{\partial\left(y^{\prime \prime}\right)^{k}}(x, 0)=x^{k \cdot \beta} \cdot \frac{\partial^{k} g}{\partial y^{k}}(x, 0) \quad \text { for all } k \in \mathbb{N} .
$$

But for $k=n-1$ we get

$$
\frac{\partial^{n-1} h}{\partial\left(y^{\prime \prime}\right)^{n-1}}(x, 0)=x^{(n-1) \cdot \beta} \cdot \frac{\partial^{n-1} g}{\partial y^{n-1}}(x, 0)=x^{n \cdot \beta} \cdot u(x)
$$

with $u(0) \neq 0$. Every partial derivative $\frac{\partial^{k} h}{\partial\left(y^{\prime \prime}\right)^{k}}(x, 0), k \geq n-1$, is thus divisible by $x^{n \cdot \beta}$. Since the quotient for $k=n-1$ is just $u(x)$ with $u(0) \neq 0$, we are able to lower the $y$-order of the function $f(x, y)$ by means of Proposition 2.7 and Lemma 2.9 applied to the functions $x^{n \cdot \beta}$ and $\frac{\partial^{k} h}{\partial\left(y^{\prime \prime}\right)^{k}}(x, 0), k=0,1, \ldots, n-2$. This completes the proof.

Repeated application of the above theorem enables us to draw the following two conclusions, which will play a crucial role in what follows. We keep the foregoing assumptions.

Proposition 3.2 (Behaviour of a Q-function at non-active infinitesimals). We can find a special cube $S=\varphi\left((0,1)^{d}\right) \subset \mathbb{R}^{m}$ with $d \leq m$, a Q-function $\omega\left(x^{\prime}, y^{\prime}\right)$ in the vicinity of $[0,1]^{d} \times \mathbb{R}$, linear with respect to the last variable and with $\omega(0,0)=0$, a Q-function $g\left(x^{\prime}, y^{\prime}\right)$ in the vicinity of $[0,1]^{d+1}$, positive infinitesimals $\lambda^{\prime}=\left(\lambda_{1}^{\prime}, \ldots, \lambda_{d}^{\prime}\right)$, an infinitesimal $\mu^{\prime}$, nonactive over $\lambda^{\prime}$, and $\alpha \in \mathbb{N}^{d}$ such that $\lambda=\varphi\left(\lambda^{\prime}\right) \in S, \mu=\omega\left(\lambda^{\prime}, \mu^{\prime}\right), g(0,0) \neq 0$ and

$$
f^{\sigma}\left(x^{\prime}, y^{\prime}\right):=f\left(\varphi\left(x^{\prime}\right), \omega\left(x^{\prime}, y^{\prime}\right)\right)=x^{\prime \alpha} g\left(x^{\prime}, y^{\prime}\right)
$$

in the vicinity of $[0,1]^{d+1}$. In particular, $v(f(\lambda, \mu))=v\left(\lambda^{\prime \alpha}\right) \in \Gamma_{\langle\lambda\rangle}$. 
Proposition 3.3 (Exchange property for a non-active infinitesimal). We can find a special cube $S=\varphi\left((0,1)^{d}\right) \subset \mathbb{R}^{m}$ with $d \leq m$, a Q-function $\omega\left(x^{\prime}, y^{\prime}\right)$ in the vicinity of $[0,1]^{d} \times \mathbb{R}$, linear with respect to the last variable and with $\omega(0,0)=0$, a Q-function $g\left(x^{\prime}, y^{\prime}\right)$ in the vicinity of $[0,1]^{d+1}$, a Q-function $h\left(x^{\prime}\right)$ in the vicinity of $[0,1]^{d}$, positive infinitesimals $\lambda^{\prime}=$ $\left(\lambda_{1}^{\prime}, \ldots, \lambda_{d}^{\prime}\right)$, an infinitesimal $\mu^{\prime}$, non-active over $\lambda^{\prime}$, and $\alpha \in \mathbb{N}^{d}$ such that $\lambda=\varphi\left(\lambda^{\prime}\right) \in S, \mu=\omega\left(\lambda^{\prime}, \mu^{\prime}\right), \frac{\partial g}{\partial y}(0,0) \neq 0$ and

$$
f^{\sigma}\left(x^{\prime}, y^{\prime}\right)-h\left(x^{\prime}\right)=f\left(\varphi\left(x^{\prime}\right), \omega\left(x^{\prime}, y^{\prime}\right)\right)-h\left(x^{\prime}\right)=x^{\prime \alpha} g\left(x^{\prime}, y^{\prime}\right)
$$

in the vicinity of $[0,1]^{d+1}$. Consequently, the non-active infinitesimal $\mu$ is the value of an $\mathcal{L}$-term $\tau(x, y)$ at the infinitesimals $\lambda$ and $\nu:=f(\lambda, \mu)$ :

$$
\mu=\tau\left(\lambda_{1}, \ldots, \lambda_{m}, \nu\right) \quad \text { or equivalently } \quad \mu \in\langle\lambda, \nu\rangle .
$$

We shall now derive some consequences of Proposition 3.2.

Corollary 3.4. Given a finite number of infinitesimals $\lambda=\left(\lambda_{1}, \ldots, \lambda_{m}\right)$, the value group $\Gamma_{\langle\lambda\rangle}$ is a vector space over $\mathbb{Q}$ of dimension $\leq m$.

We argue by induction on the number $m$ of generators. We may, of course, assume that the infinitesimals $\lambda$ are analytically independent. It suffices to show that the vector space spanned by the set

$$
\left\{v(f(\lambda)): f \text { is a Q-function at } 0 \in \mathbb{R}^{m}\right\}
$$

is of dimension $d(\lambda) \leq m$. Indeed, supposing the vectors $v\left(t_{1}(\lambda)\right), \ldots$, $v\left(t_{m+1}(\lambda)\right)$ to be linearly independent over $\mathbb{Q}$, by applying $m+1$ times Corollary 2.6 we would find infinitesimals $\lambda^{\prime}=\left(\lambda_{1}^{\prime}, \ldots, \lambda_{m}^{\prime}\right) \in\langle\lambda\rangle$ and Q-functions $f_{1}(x), \ldots, f_{m+1}(x)$ at $0 \in \mathbb{R}^{m}$ such that

$$
t_{i}(\lambda)=f_{i}\left(\lambda^{\prime}\right) \text { for } i=1, \ldots, m+1 .
$$

Hence we would get $d\left(\lambda^{\prime}\right)>m$, which contradicts our hypothesis.

Suppose now that the assertion holds for $m$ and take $m+1$ infinitesimals $\lambda=\left(\lambda_{1}, \ldots, \lambda_{m}\right), \mu$. We must show that $d(\lambda, \mu) \leq m+1$. If $\mu$ is non-active over $\lambda$, it follows from Proposition 3.2 and the induction hypothesis that

$$
d(\lambda, \mu)=d(\lambda) \leq \operatorname{dim} \Gamma_{\langle\lambda\rangle} \leq m .
$$

In the other case, $v(\mu-t(\lambda)) \notin \Gamma_{\langle\lambda\rangle}$ for an $\mathcal{L}$-term $t(x)$. By the desingularization of $\mathcal{L}$-terms (Corollary 2.6), one can find a special modification $\varphi$ and infinitesimals $\lambda^{\prime}$ such that $\lambda=\varphi\left(\lambda^{\prime}\right)$ and $f:=t \circ \varphi$ is a Q-function at $0 \in \mathbb{R}^{m}$. Then $t(\lambda)=f\left(\lambda^{\prime}\right)$ and $v\left(\mu-f\left(\lambda^{\prime}\right)\right) \notin \Gamma_{\langle\lambda\rangle}$. Consequently, from Corollary 2.11 and the induction hypothesis we get the inequalities

$$
d(\lambda, \mu) \leq d\left(\lambda^{\prime}, \mu\right)=d\left(\lambda^{\prime}\right)+1 \leq \operatorname{dim} \Gamma_{\left\langle\lambda^{\prime}\right\rangle}+1 \leq m+1,
$$

which is the desired result. 
Proposition 3.5 (Behaviour of an $\mathcal{L}$-term at non-active infinitesimals). For each $\mathcal{L}$-term $t(x, y)$, there exist a special modification $\varphi:(0,1)^{d} \rightarrow \mathbb{R}^{m}$ with $d \leq m$, a Q-function $\omega\left(x^{\prime}, y^{\prime}\right)$ in the vicinity of $[0,1]^{d} \times \mathbb{R}$, linear with respect to the last variable and with $\omega(0)=0$, a Q-function $f\left(x^{\prime}, y^{\prime}\right)$ in the vicinity of $[0,1]^{d+1}$, positive infinitesimals $\lambda^{\prime}=\left(\lambda_{1}^{\prime}, \ldots, \lambda_{d}^{\prime}\right)$ and a positive infinitesimal $\mu^{\prime}$, non-active over $\lambda^{\prime}$, such that $\lambda=\varphi\left(\lambda^{\prime}\right) \in S, \mu=\omega\left(\lambda^{\prime}, \mu^{\prime}\right)$ and for all $\left(x^{\prime}, y^{\prime}\right) \in(0,1)^{d+1}$ we have

$$
t^{\sigma}\left(x^{\prime}, y^{\prime}\right)=f\left(x^{\prime}, y^{\prime}\right) \quad \text { or } \quad t^{\sigma}\left(x^{\prime}, y^{\prime}\right)=\frac{1}{f\left(x^{\prime}, y^{\prime}\right)},
$$

according as $t(\lambda, \mu)$ is bounded or not; here $t^{\sigma}\left(x^{\prime}, y^{\prime}\right):=t\left(\varphi\left(x^{\prime}\right), \omega\left(x^{\prime}, y^{\prime}\right)\right)$. In particular, we have the dichotomy: either

- an infinitesimal $\mu$ is active over $\lambda$, or

- $\Gamma_{\langle\lambda, \mu\rangle}=\Gamma_{\langle\lambda\rangle}$.

The proof is by induction on the complexity of the term $t(x, y)$ with substitution of a special modification, and consists in repeated application of Proposition 3.2 and transformation to normal crossings of the functions in the non-distinguished variables $x^{\prime}$, which occur in the process. In the case of a product of two $\mathcal{L}$-terms, one should simultaneously transform to normal crossings the two functions in the non-distinguished variables $x^{\prime}$, so that the two exponents obtained are totally ordered (as in the proof of Proposition 2.7). In the case of a root of an $\mathcal{L}$-term, after transformation to normal crossings, one should substitute suitable power functions. The detailed verification is straightforward, and we leave it to the reader. Note, however, that the equality in question holds only for $\left(x^{\prime}, y^{\prime}\right) \in(0,1)^{d+1}$, and not in the vicinity of $[0,1]^{d+1}$.

Propositions 3.3 and 3.5 immediately yield the following

COROLLARY 3.6 (Exchange property for a non-active infinitesimal). We can find a special modification $\varphi:(0,1)^{d} \rightarrow \mathbb{R}^{m}$ with $d \leq m$, a Q-function $\omega\left(x^{\prime}, y^{\prime}\right)$ in the vicinity of $[0,1]^{d} \times \mathbb{R}$, linear with respect to the last variable and with $\omega(0,0)=0$, a Q-function $g\left(x^{\prime}, y^{\prime}\right)$ in the vicinity of $[0,1]^{d+1}$, a Q-function $h\left(x^{\prime}\right)$ in the vicinity of $[0,1]^{d}$, positive infinitesimals $\lambda^{\prime}=$ $\left(\lambda_{1}^{\prime}, \ldots, \lambda_{d}^{\prime}\right)$, an infinitesimal $\mu^{\prime}$, non-active over $\lambda^{\prime}$, and $\alpha \in \mathbb{N}^{m}$ such that $\lambda=\varphi\left(\lambda^{\prime}\right) \in S, \mu=\omega\left(\lambda^{\prime}, \mu^{\prime}\right), \frac{\partial g}{\partial y}(0,0) \neq 0$ and

$$
t^{\sigma}\left(x^{\prime}, y^{\prime}\right)-h\left(x^{\prime}\right)=t\left(\varphi\left(x^{\prime}\right), \omega\left(x^{\prime}, y^{\prime}\right)\right)-h\left(x^{\prime}\right)=x^{\prime \alpha} g\left(x^{\prime}, y^{\prime}\right)
$$

in the vicinity of $[0,1]^{d+1}$. Consequently, the non-active infinitesimal $\mu$ is the value of an $\mathcal{L}$-term $\tau(x, y)$ at the infinitesimals $\lambda$ and $\nu:=t(\lambda, \mu)$ :

$$
\mu=\tau\left(\lambda_{1}, \ldots, \lambda_{m}, \nu\right) \quad \text { or equivalently } \quad \mu \in\langle\lambda, \nu\rangle .
$$


4. Valuation property for $\mathcal{L}$-terms. We begin with

Proposition 4.1. Consider a sequence $\lambda=\left(\lambda_{1}, \ldots, \lambda_{k}\right)$ of positive infinitesimals whose valuations are linearly independent over $\mathbb{Q}$. Then, for each term $t\left(x_{1}, \ldots, x_{k}\right)$ with $t(\lambda)$ bounded, there are a $Q$-function $f$ at $0 \in \mathbb{R}^{k}$ and multi-indices $\beta_{i}=\left(\beta_{i 1}, \ldots, \beta_{i k}\right) \in \mathbb{Q}^{k}, i=1, \ldots, k$, linearly independent over $\mathbb{Q}$, such that $\lambda^{\beta_{1}}, \ldots, \lambda^{\beta_{m}}$ are positive infinitesimals and

$$
t(\lambda)=f\left(\lambda^{\beta_{1}}, \ldots, \lambda^{\beta_{k}}\right) .
$$

Our proof starts with the observation that, due to the desingularization of $\mathcal{L}$-terms (Corollary 2.6), there exist a special cube $S=\varphi\left((0,1)^{k}\right) \subset \mathbb{R}^{k}$, a Q-function $g\left(x^{\prime}\right)$ in the vicinity of $[0,1]^{m}$, positive infinitesimals $\lambda^{\prime}=$ $\left(\lambda_{1}^{\prime}, \ldots, \lambda_{k}^{\prime}\right)$ such that $\lambda=\varphi\left(\lambda^{\prime}\right) \in S$ and

$$
t^{\varphi}\left(x^{\prime}\right):=t\left(\varphi\left(x^{\prime}\right)\right)=g\left(x^{\prime}\right) \quad \text { for all } x^{\prime} \in(0,1)^{k} .
$$

Via transformation to normal crossings by blowing up, applied to the functions $\varphi_{1}, \ldots, \varphi_{k}$ and the coordinate functions $x_{1}, \ldots, x_{k}$, one can assume that the infinitesimals $\lambda^{\prime}$ are a regular sequence too, and that

$$
\varphi_{i}\left(x^{\prime}\right)=u_{i}\left(x^{\prime}\right)\left(x^{\prime}\right)^{\alpha_{i}} \quad \text { with } \alpha_{i}=\left(\alpha_{i 1}, \ldots, \alpha_{i k}\right) \in \mathbb{N}^{k}, i=1, \ldots, k,
$$

where the Q-functions $u_{i}\left(x^{\prime}\right)$ are units at zero and the multi-indices $\alpha_{i}$, $i=1, \ldots, k$, are linearly independent over $\mathbb{Q}$. Let $A$ be the matrix whose rows are just the multi-indices $\alpha_{i}$, and $B$ the inverse matrix with rows $\beta_{l}=$ $\left(\beta_{l 1}, \ldots, \beta_{l k}\right) \in \mathbb{Q}^{k}$. Consider the mapping

$$
\psi:(0, \infty)^{k} \rightarrow(0, \infty)^{k}, \quad \psi(x):=\left(x^{\beta_{1}}, \ldots, x^{\beta_{k}}\right) .
$$

Then the superposition $\chi=\left(\chi_{1}, \ldots, \chi_{k}\right):=\psi \circ \varphi$ is of the form $\chi_{i}\left(x^{\prime}\right)=$ $x_{i}^{\prime} v_{i}\left(x^{\prime}\right), i=1, \ldots, k$, where the Q-functions $v_{i}\left(x^{\prime}\right)$ are units at zero. Hence

$$
\lambda^{\beta_{1}}=\chi_{1}\left(\lambda^{\prime}\right), \ldots, \lambda^{\beta_{k}}=\chi_{k}\left(\lambda^{\prime}\right)
$$

are infinitesimals, and the mapping $\chi$ is invertible; put $\omega:=\chi^{-1}$. Then $x^{\prime}=\omega\left(x^{\beta_{1}}, \ldots, x^{\beta_{k}}\right)$, and thus we get

$$
t(\lambda)=g\left(\lambda^{\prime}\right)=(g \circ \omega)\left(\lambda^{\beta_{1}}, \ldots, \lambda^{\beta_{k}}\right) .
$$

Putting $f:=g \circ \omega$ finishes the proof.

We now introduce the following definition: a sequence $\lambda=\left(\lambda_{1}, \ldots, \lambda_{m}\right)$ of infinitesimals will be called regular with main part $\lambda_{1}, \ldots, \lambda_{k}$ if the valuations

$$
v\left(\lambda_{1}\right), \ldots, v\left(\lambda_{k}\right) \in \Gamma_{\langle\lambda\rangle}
$$

form a basis over $\mathbb{Q}$ of the valuation group $\Gamma_{\left\langle\lambda_{1}, \ldots, \lambda_{m}\right\rangle}$. Note that $k \leq m$ by Corollary 3.4.

Combining Proposition 4.1 with Proposition 3.5 (on behaviour of an $\mathcal{L}$ term at non-active infinitesimals), we immediately obtain 
Corollary 4.2 (Behaviour of an $\mathcal{L}$-term at a regular sequence). Suppose that $\lambda=\left(\lambda_{1}, \ldots, \lambda_{m}\right)$ is a regular sequence of positive infinitesimals with main part $\lambda_{1}, \ldots, \lambda_{k}, k<m$. Each infinitesimal $\lambda_{j}, j=k+1, \ldots, m$, is obviously non-active over the preceding infinitesimals. Then, for each $\mathcal{L}$ term $t\left(x_{1}, \ldots, x_{m}\right)$ with $t(\lambda)$ bounded, there are a $Q$-function $f$ at $0 \in \mathbb{R}^{m}$ and multi-indices $\beta_{i}=\left(\beta_{i 1}, \ldots, \beta_{i k}\right) \in \mathbb{Q}^{k}, i=1, \ldots, k$, linearly independent over $\mathbb{Q}$, such that $\left(\lambda_{1}, \ldots, \lambda_{k}\right)^{\beta_{1}}, \ldots,\left(\lambda_{1}, \ldots, \lambda_{k}\right)^{\beta_{m}}$ are positive infinitesimals and

$$
t(\lambda)=f\left(\left(\lambda_{1}, \ldots, \lambda_{k}\right)^{\beta_{1}}, \ldots,\left(\lambda_{1}, \ldots, \lambda_{k}\right)^{\beta_{k}}, \lambda_{k+1}, \ldots, \lambda_{m}\right) .
$$

In particular, the value of any $\mathcal{L}$-term at a regular sequence of infinitesimals coincides with the value of a $Q$-function at a regular sequence of infinitesimals that generates the same $\mathcal{L}$-substructure.

We are now in a position to prove a basic theorem on an active infinitesimal, stated below. For the analytic case, we shall present a direct proof which makes use of diagonal series. However, in the general quasianalytic settings, we are not able to apply this technique as yet, because we do not know whether the diagonal series of a quasianalytic function determines a quasianalytic function. Our further deduction is as follows: we derive from this theorem the valuation and exchange properties for $\mathcal{L}$-terms; next, in Section 5, quantifier elimination for the theory $T$ under study is established.

REMARK 4.3. We are able to provide a complete proof of the theorem below for the particular quasianalytic case where the valuations of the infinitesimals $\lambda_{1}, \ldots, \lambda_{m}$ are linearly independent over $\mathbb{Q}$. The proof is quite complicated. Instead of diagonal series, we use a recursive method of admissible annihilation of a sufficient yet finite number of diagonal Taylor coefficients of the function under study. An affirmative answer to the problem of diagonal series for the general quasianalytic case would allow us to extend verbatim our proof for the analytic case to the general quasianalytic settings.

THEOREM 4.4 (on an active infinitesimal). Consider a regular sequence $\mu, \lambda_{1}, \ldots, \lambda_{m}$ of infinitesimals with main part $\mu, \lambda_{1}, \ldots, \lambda_{k}$ and an $\mathcal{L}$-term $t(y, x), x=\left(x_{1} \ldots, x_{m}\right)$, such that

$$
\nu:=t(\mu, \lambda) \notin\langle\lambda\rangle
$$

is an infinitesimal. Then $\nu$ is active over the infinitesimals $\lambda$.

Direct proof for the analytic case. We first reduce the form taken by the term $t(y, x)$ at the infinitesimals $(\mu, \lambda)$. We may, of course, assume that all the infinitesimals under consideration are positive. Put

$$
\lambda^{\prime}=\left(\lambda_{1}, \ldots, \lambda_{k}\right), \quad \lambda^{\prime \prime}=\left(\lambda_{k+1}, \ldots, \lambda_{m}\right) ;
$$


by Proposition 4.1 we have

$$
\nu=t(\mu, \lambda)=f\left(\left(\lambda^{\prime}, \mu\right)^{\beta_{1}}, \ldots,\left(\lambda^{\prime}, \mu\right)^{\beta_{k+1}}, \lambda^{\prime \prime}\right)
$$

for some Q-function $f$ at $0 \in \mathbb{R}^{m+1}$ and multi-indices

$$
\beta_{i}=\left(\beta_{i, 1}, \ldots, \beta_{i, k+1}\right) \in \mathbb{Q}^{k+1}, \quad i=1, \ldots, k+1,
$$

linearly independent over $\mathbb{Q}$, such that $\left(\lambda^{\prime}, \mu\right)^{\beta_{1}}, \ldots,\left(\lambda^{\prime}, \mu\right)^{\beta_{k+1}}$ are positive infinitesimals. Without loss of generality, the problem can be reduced to the case where $\beta_{i}=\left(\beta_{i, 1}, \ldots, \beta_{i, k+1}\right) \in \mathbb{Z}^{k+1}, i=1, \ldots, k+1$, and next to the case

$$
\nu=f\left(\left(\lambda^{\prime}\right)^{\alpha_{1}} \mu^{\epsilon_{1}}, \ldots,\left(\lambda^{\prime}\right)^{\alpha_{k+1}} \mu^{\epsilon_{k+1}}, \lambda^{\prime \prime}\right)
$$

with $\epsilon_{i} \in\{-1,0,1\}, i=1, \ldots, k+1$.

But one can always replace a Q-function $f(u, v)$ by $g(u, v / u)$, where the Q-function $g\left(u^{\prime}, v^{\prime}\right)$ is given by the formula $g\left(u^{\prime}, v^{\prime}\right):=f\left(u^{\prime}, u^{\prime} v^{\prime}\right)$. Since the valuations of the infinitesimals $\left(\lambda^{\prime}\right)^{\alpha_{1}} \mu^{\epsilon_{1}}, \ldots,\left(\lambda^{\prime}\right)^{\alpha_{k+1}} \mu^{\epsilon_{k+1}}$ are pairwise distinct, we can thus reduce the situation to the case where

$$
\nu=f\left(\left(\lambda^{\prime}\right)^{\alpha_{1}} \mu,\left(\lambda^{\prime}\right)^{\alpha_{2}} / \mu,\left(\lambda^{\prime}\right)^{\alpha_{3}}, \ldots,\left(\lambda^{\prime}\right)^{\alpha_{k+1}}, \lambda^{\prime \prime}\right),
$$

and next to the case

$$
\nu=f\left(\mu,\left(\lambda^{\prime}\right)^{\alpha_{1}} / \mu,\left(\lambda^{\prime}\right)^{\alpha_{2}}, \ldots,\left(\lambda^{\prime}\right)^{\alpha_{k}}, \lambda^{\prime \prime}\right) .
$$

Since the multi-indices $\alpha_{1}, \ldots, \alpha_{k} \in \mathbb{Z}^{k}$ are obviously linearly independent over $\mathbb{Q}$, we can eventually assume, without loss of generality, that

$$
\nu=f\left(\mu, \lambda_{1} / \mu, \widetilde{\lambda}\right) \text { with } \tilde{\lambda}=\left(\lambda_{2} \ldots, \lambda_{k}, \lambda_{k+1}, \ldots, \lambda_{m}\right),
$$

where $f(u, v, \widetilde{x})$ is a Q-function at $0 \in \mathbb{R}^{m+1}$ and $\widetilde{x}=\left(x_{2}, \ldots, x_{m}\right)$.

But in the analytic case we certainly have at our disposal diagonal decompositions of Taylor series. Therefore we can write the function $f$ as follows:

$$
f(u, v, \widetilde{x})=u \cdot g(u, u v, \widetilde{x})+v \cdot h(v, u v, \widetilde{x})+\varphi(u v, \widetilde{x}),
$$

where $g, h$ are Q-functions at $0 \in \mathbb{R}^{m+1}$ and $\varphi$ is a Q-function at $0 \in \mathbb{R}^{m}$. Hence

$$
\begin{aligned}
\nu & =\mu \cdot g\left(\mu, \lambda_{1}, \widetilde{\lambda}\right)+\lambda_{1} / \mu \cdot h\left(\lambda_{1} / \mu, \lambda_{1}, \widetilde{\lambda}\right)+\varphi\left(\lambda_{1}, \widetilde{\lambda}\right) \\
& =\mu \cdot g(\mu, \lambda)+\lambda_{1} / \mu \cdot h\left(\lambda_{1} / \mu, \lambda\right)+\varphi(\lambda) .
\end{aligned}
$$

Since $\nu=f\left(\mu, \lambda_{1} / \mu, \widetilde{\lambda}\right) \notin\langle\lambda\rangle$, the functions $g$ and $h$ cannot vanish simultaneously: $g \not \equiv 0$ or $h \not \equiv 0$. It follows immediately from Corollary 2.11 that the valuations of the infinitesimals

$$
\mu \cdot g\left(\mu, \lambda_{1}, \widetilde{\lambda}\right) \text { and } \quad \lambda_{1} / \mu \cdot h\left(\lambda_{1} / \mu, \lambda_{1}, \widetilde{\lambda}\right)
$$

lie respectively in

$$
\Gamma_{\left\langle\lambda_{1}, \ldots, \lambda_{m}\right\rangle}+\mathbb{Z}_{+} \cdot v(\mu) \text { and } \Gamma_{\left\langle\lambda_{1}, \ldots, \lambda_{m}\right\rangle}+\mathbb{Z}_{-} \cdot v(\mu) .
$$


Consequently, these valuations are distinct and do not belong to the group $\Gamma_{\left\langle\lambda_{1}, \ldots, \lambda_{m}\right\rangle}$, and thus we get $v(\nu-\varphi(\lambda))=\min \left\{v\left(\mu \cdot g\left(\mu, \lambda_{1}, \tilde{\lambda}\right)\right), v\left(\lambda_{1} / \mu \cdot h\left(\lambda_{1} / \mu, \lambda_{1}, \widetilde{\lambda}\right)\right)\right\} \notin \Gamma_{\left\langle\lambda_{1}, \ldots, \lambda_{m}\right\rangle}$.

This means that $\nu$ is active over the infinitesimals $\lambda$, as asserted.

Proof for a particular quasianalytic case. We assume in addition that the valuations of the infinitesimals $\lambda_{1}, \ldots, \lambda_{m}$ are independent over $\mathbb{Q}$. As before, we are reduced to the case where

$$
\nu=f\left(\mu, \lambda_{1} / \mu, \tilde{\lambda}\right) \quad \text { with } \tilde{\lambda}=\left(\lambda_{2}, \ldots, \lambda_{m}\right),
$$

where $f(u, v, \widetilde{x})$ is a Q-function at $0 \in \mathbb{R}^{m+1}$ and $\widetilde{x}=\left(x_{2}, \ldots, x_{m}\right)$. Let

$$
\widehat{f}(u, v, \widetilde{x})=\sum_{i, j \in \mathbb{N}} \sum_{\gamma \in \mathbb{N}^{m-1}} a_{i, j, \gamma} \cdot u^{i} v^{j} \widetilde{x}^{\gamma}=\sum_{i, j \in \mathbb{N}} a_{i, j}(\widetilde{x}) \cdot u^{i} v^{j}
$$

be the Taylor series at zero of the function $f(u, v, \widetilde{x})$. First observe that not all of the coefficients $a_{i, j, \gamma}$ with $i, j \in \mathbb{N}, i \neq j, \gamma \in \mathbb{N}^{m-1}$, of the Taylor series at zero of the function $f(u, v, \widetilde{x})$ vanish. This follows immediately from the lemma below, since otherwise we would get

$$
\nu=f\left(\mu, \lambda_{1} / \mu, \widetilde{\lambda}\right)=h\left(\lambda_{1}, \widetilde{\lambda}\right)=h(\lambda) \in\langle\lambda\rangle,
$$

contrary to the assumption of our theorem.

LEMMA 4.5. With the above notation, if all the Taylor coefficients at zero $a_{i, j, \gamma}$ with $i, j \in \mathbb{N}, i \neq j, \gamma \in \mathbb{N}^{m-1}$, of a Q-function $f(u, v, \widetilde{x})$ vanish, then

$$
f(u, v, \widetilde{x})=h(u v, \widetilde{x})
$$

for a $Q$-function $h(w, \widetilde{x})$ at zero.

In order to prove this, fix $u$ and $v$, and consider the Q-function

$$
h_{u, v}(t, \widetilde{x}):=f(t u, t v, \widetilde{x}) .
$$

It is easy to check that the Taylor series at zero of the functions $h_{u, v}(t, \widetilde{x})$ coincide whenever $u v=w$. By quasi-analyticity, the functions $h_{u, v}(t, \widetilde{x})$ coincide whenever $u v=w$. Hence $f(u, v, \widetilde{x})=h(u v, \widetilde{x})$ for a definable function $h(w, \widetilde{x})$ near zero. When we fix a sufficiently small $u \neq 0$ from the source of the function $f$, we have

$$
h(w, \widetilde{x})=f(u, w / u, \widetilde{x}),
$$

which demonstrates that $h(w, \widetilde{x})$ is a Q-function at zero, as required.

Now we wish to repeat the method from the proofs of Proposition 2.7 and its corollaries, which consists in a simultaneous transformation to normal crossings of some partial derivatives of a given Q-function so as to get finitely many exponents totally ordered with respect to the induced partial ordering from $\mathbb{N}^{m}$. It uses the noetherianity of the rings of formal power series and the 
fact that a Q-function is divisible by a monomial whenever so is its Taylor series (see Remark 1.1(3)). We apply this method to the partial derivatives

$$
\frac{\partial^{i+j} f}{\partial u^{i} \partial v^{j}}(0,0, \widetilde{x}), \quad i, j \in \mathbb{N}, i \neq j .
$$

In this fashion one can find a special cube $S=\varphi\left((0,1)^{m-1}\right) \subset \mathbb{R}^{m-1}$ with $\varphi$ being a composite of successive blowings-up, and positive infinitesimals $\widetilde{\lambda}^{\prime}=\left(\lambda_{2}^{\prime}, \ldots, \lambda_{m}^{\prime}\right)$ such that

$$
\widetilde{\lambda}=\varphi\left(\widetilde{\lambda}^{\prime}\right) \in S
$$

and the partial derivatives $\frac{\partial^{i+j}}{\partial u^{i} \partial v^{j}}\left(0,0, \widetilde{x}^{\prime}\right)$ with $i \neq j$ of the function

$$
f^{\varphi}\left(u, v, \widetilde{x}^{\prime}\right):=f\left(\varphi\left(u, v, \widetilde{x}^{\prime}\right)\right)
$$

are divisible by a certain partial derivative

$$
\frac{\partial^{p+q} f^{\varphi}}{\partial u^{p} \partial v^{q}}\left(0,0, \widetilde{x}^{\prime}\right)=\left(\widetilde{x}^{\prime}\right)^{\alpha} \cdot \operatorname{unit}\left(\widetilde{x}^{\prime}\right) \quad \text { with } p \neq q, \alpha \in \mathbb{N}^{m-1},
$$

which is a normal crossing. One can, of course, replace the infinitesimals $\lambda_{2}, \ldots, \lambda_{m}$ with the new ones $\lambda_{2}^{\prime}, \ldots, \lambda_{m}^{\prime}$ and the function $f$ with $f^{\varphi}$. For simplicity we drop the prime on $\widetilde{x}$.

We shall have proved that the infinitesimal $\nu=f\left(\mu, \lambda_{1} / \mu, \widetilde{\lambda}\right)$ is active over the infinitesimals $\lambda$ if we find a Q-function $g(w, \widetilde{x})$ at $0 \in \mathbb{R}^{m}$ such that

$$
f\left(\mu, \lambda_{1} / \mu, \widetilde{\lambda}\right)-g\left(\lambda_{1}, \widetilde{\lambda}\right)=f\left(\mu, \lambda_{1} / \mu, \widetilde{\lambda}\right)-g(\lambda) \notin \Gamma_{\langle\lambda\rangle} \cdot
$$

A function $g(w, \widetilde{x})$ as above will be constructed recursively as follows. We first find a finite sequence of Q-functions $g_{l}(w, \widetilde{x}), f_{l}(u, v, \widetilde{x})$ at zero, and of multi-indices $\delta_{l} \in \mathbb{N}^{m-1}, 1 \leq l \leq L$ with $L \leq|\alpha|$, such that

$$
\begin{array}{r}
f(u, v, \widetilde{x})-g_{1}(u v, \widetilde{x})=\widetilde{x}^{\delta_{1}} \cdot f_{1}(u, v, \widetilde{x}), \\
f_{1}(u, v, \widetilde{x})-g_{2}(u v, \widetilde{x})=\widetilde{x}^{\delta_{2}} \cdot f_{2}(u, v, \widetilde{x}), \\
f_{L-1}(u, v, \widetilde{x})-g_{L}(u v, \widetilde{x})=\widetilde{x}^{\delta_{L}} \cdot f_{L}(u, v, \widetilde{x}),
\end{array}
$$

where the partial derivative $\frac{\partial^{p+q} f_{L}}{\partial u^{p} \partial v^{q}}(0,0, \widetilde{x})$ is a Q-analytic unit at zero.

In order to construct the functions $g_{l}(w, \widetilde{x}), f_{l}(u, v, \widetilde{x})$, we start from $f_{0}(u, v, \widetilde{x}):=f(u, v, \widetilde{x})$ and suppose that $x_{m}$ occurs in $x^{\alpha}$ with a positive exponent. Then the Taylor series of the function

$$
f_{0}\left(u, v, x_{2}, \ldots, x_{m-1}, 0\right)
$$

has non-zero coefficients

$$
b_{i, j, \beta_{2}, \ldots, \beta_{m-1}} \cdot u^{i} v^{j} x_{2}^{\beta_{2}} \cdots x_{m-1}^{\beta_{m-1}}
$$

only for $i=j$ (i.e. on the diagonal with respect to the variables $u, v$ ), and thus, according to Lemma 1, we get 


$$
f_{0}\left(u, v, 0, x_{2}, \ldots, x_{m-1}, 0\right)=g\left(u v, x_{2}, \ldots, x_{m-1}\right)
$$

for a Q-function $g\left(w, x_{2}, \ldots, x_{m-1}\right)$ at zero. Moreover, the difference

$$
f_{0}(u, v, \widetilde{x})-g\left(u v, x_{2}, \ldots, x_{m-1}\right)
$$

is divisible by a positive power $x_{m}^{\delta_{m}}$ (see Remark 1.1(3)), whence

$$
f_{0}(u, v, \widetilde{x})-g\left(u v, x_{2}, \ldots, x_{m-1}\right)=x_{m}^{\delta_{m}} f_{1}(u, v, \widetilde{x})
$$

for a Q-function $f_{1}(u, v, \widetilde{x})$. It is easy to check that

$$
\frac{\partial^{p+q} f_{1}}{\partial u^{p} \partial v^{q}}(0,0, \widetilde{x})=\widetilde{x}^{\alpha_{1}} \cdot \operatorname{unit}(\widetilde{x})
$$

with $\alpha_{1}:=\alpha-\left(0, \ldots, 0, \delta_{m}\right),\left|\alpha_{1}\right|<|\alpha|$. We proceed further by descending induction on $\left|\alpha_{l}\right|$ where

$$
\frac{\partial^{p+q} f_{l}^{\varphi}}{\partial u^{p} \partial v^{q}}(0,0, \widetilde{x})=\widetilde{x}^{\alpha_{l}} \cdot \operatorname{unit}(\widetilde{x}) .
$$

Having constructed the above finite sequences of functions, it is sufficient to construct the desired function $g(w, \widetilde{x})$ for the last function $f_{L}(u, v, \widetilde{x})$, and thus we are reduced to the case where a partial derivative

$$
\frac{\partial^{p+q} f}{\partial u^{p} \partial v^{q}}(0,0, \widetilde{x}) \quad \text { with } p \neq q
$$

is a Q-analytic unit at zero. We now show that the function

$$
g(w, \widetilde{x}):=\sum_{i=0}^{\max \{p, q\}} \frac{1}{(i !)^{2}} \cdot \frac{\partial^{2 i} f}{\partial u^{i} \partial v^{i}}(0,0, \widetilde{x}) \cdot w^{i}
$$

satisfies the desired condition, i.e.

$$
\eta\left(\mu, \lambda_{1} / \mu, \widetilde{\lambda}\right) \notin \Gamma_{\langle\lambda\rangle} \quad \text { for the function } \quad \eta(u, v, \widetilde{x}):=f(u, v, \widetilde{x})-g(u v, \widetilde{x}) .
$$

This follows immediately from the lemma below, because its assumptions are obviously satisfied by the above function $\eta(u, v, \widetilde{x})$.

LEMma 4.6. If $\eta(u, v, \widetilde{x})$ is a $Q$-function at zero with Taylor coefficients $c_{i, j, \gamma}$ such that $c_{p, q, 0, \ldots, 0} \neq 0$ for some $p, q \in \mathbb{N}$ with $p \neq q$, and $c_{i, i, \gamma}=0$ for all $\gamma \in \mathbb{N}^{m-1}$ and $i \in \mathbb{N}$ with $i \leq \max \{p, q\}$, then $\eta\left(\mu, \lambda_{1} / \mu, \widetilde{\lambda}\right) \notin \Gamma_{\langle\lambda\rangle}$.

In order to prove this, we shall once again use Axiom 5 on the quasianalytic class $\mathrm{Q}$ to the effect that $\mathrm{Q}$ is closed under division by a coordinate (see also Remark 1.1(3)). Hence we have

$$
\eta(u, v, \widetilde{x})-\sum_{j=0}^{q-1} \frac{\partial^{j} \eta}{\partial v^{j}}(u, 0, \widetilde{x}) \cdot \frac{v^{j}}{j !}=v^{q} \cdot \zeta(u, v, \widetilde{x})
$$


for some Q-function $\zeta(u, v, \widetilde{x})$ at zero, and

$$
\zeta(u, v, \widetilde{x})-\sum_{i=0}^{p-1} \frac{\partial^{i} \zeta}{\partial u^{i}}(0, v, \widetilde{x}) \cdot \frac{u^{i}}{i !}=u^{p} \cdot \omega(u, v, \widetilde{x})
$$

for some $\mathrm{Q}$-function $\omega(u, v, \widetilde{x})$ at zero.

Again, through repeating successive modifications of the variables $\widetilde{x}$ by means of special cubes, as in Corollary 2.10, one can assume that

$$
\frac{1}{j !} \cdot \frac{\partial^{j} \eta}{\partial v^{j}}(u, 0, \widetilde{x})=\widetilde{x}^{\gamma_{j}} \cdot \eta_{j}(u, \widetilde{x})
$$

where $\eta_{j}(u, \widetilde{x}), j=0, \ldots, q-1$, are $u$-regular Q-functions at zero, say, of order $r_{j}$; similarly,

$$
\frac{1}{i !} \cdot \frac{\partial^{i} \zeta}{\partial u^{i}}(0, v, \widetilde{x})=\widetilde{x}^{\delta_{i}} \cdot \zeta_{i}(v, \widetilde{x}),
$$

where $\zeta_{i}(v, \widetilde{x}), i=0, \ldots, p-1$, are $v$-regular Q-functions at zero, say, of order $s_{i}$. Therefore we are able to write down these functions in the following form (cf. Corollary 2.11):

$$
\eta_{j}(u, \widetilde{x})=\sum_{r=0}^{r_{j}-1} \eta_{j, r}(\widetilde{x}) \cdot u^{r}+\eta_{j, r_{j}}(u, \widetilde{x}) \cdot u^{r_{j}} \quad \text { with } \eta_{j, r_{j}}(0,0) \neq 0
$$

and

$$
\zeta_{i}(v, \widetilde{x})=\sum_{s=0}^{s_{i}-1} \zeta_{i, s}(\widetilde{x}) \cdot v^{s}+\zeta_{i, s_{i}}(v, \widetilde{x}) \cdot v^{s_{i}} \quad \text { with } \zeta_{i, s_{i}}(0,0) \neq 0
$$

all functions which occur above are Q-analytic at zero.

Moreover, making use of transformation to normal crossings by blowing up, one can also assume that

$$
\eta_{j, r}(\widetilde{x})=\widetilde{x}^{\alpha_{j, r}} \cdot \operatorname{unit}(\widetilde{x}) \quad \text { and } \quad \zeta_{i, s}(\widetilde{x})=\widetilde{x}^{\beta_{i, s}} \cdot \operatorname{unit}(\widetilde{x}),
$$

with some $\alpha_{j, r}, \beta_{i, s} \in \mathbb{N}^{m-1}$.

Then

$$
\begin{aligned}
\eta(u, v, \widetilde{x}) & =\sum_{j=0}^{q-1} v^{j}\left[\sum_{r=0}^{r_{j}-1} u^{r} \widetilde{x}^{\gamma_{j}} \cdot \eta_{j, r}(\widetilde{x})+u^{r_{j}} \widetilde{x}^{\gamma_{j}} \cdot \operatorname{unit}(u, \widetilde{x})\right] \\
& +\sum_{i=0}^{p-1} u^{i} v^{q}\left[\sum_{s=0}^{s_{i}-1} v^{s} \widetilde{x}^{\delta_{i}} \cdot \zeta_{i, s}(\widetilde{x})+v^{s_{i}} \widetilde{x}^{\delta_{i}} \cdot \operatorname{unit}(v, \widetilde{x})\right]+u^{p} v^{q} \cdot \omega(\widetilde{x}, u, v) .
\end{aligned}
$$

Since $\frac{\partial^{p+q} \eta}{\partial u^{p} \partial v^{q}}(0,0,0) \neq 0$, we get $\omega(0,0,0) \neq 0$. Substituting

$$
\mu, \lambda_{1} / \mu, \lambda_{2}, \ldots, \lambda_{m} \quad \text { for } \quad u, v, x_{2}, \ldots, x_{m}
$$


and taking into account our additional assumption about the infinitesimals $\lambda$, we deduce that the valuations of the summands in the above formula are pairwise distinct. Consequently, $v\left(\eta\left(\mu, \lambda_{1} / \mu, \widetilde{\lambda}\right)\right)$ coincides with the valuation of one summand in the above formula.

But our assumption about the vanishing of certain coefficients (lying on the diagonal with respect to the variables $u, v$ ) of the Taylor series of the function $\eta(u, v, \widetilde{x})$ implies that no summand with a factor of the form $u^{i} v^{i}$ occurs in the above summation. It follows immediately that

$$
v\left(\eta\left(\mu, \lambda_{1} / \mu, \tilde{\lambda}\right)\right) \in v\left(\mu^{i} \cdot\left(\lambda_{m} / \mu\right)^{j}\right)+\Gamma_{\langle\lambda\rangle}=v\left(\mu^{i-j}\right)+\Gamma_{\langle\lambda\rangle}
$$

for some $i, j \in \mathbb{N}, i \neq j$. Hence $v\left(\eta\left(\mu, \lambda_{1} / \mu, \widetilde{\lambda}\right)\right) \notin \Gamma_{\langle\lambda\rangle}$, which proves Lemma 4.6.

We have thus shown that

$$
v(\nu-g(\lambda))=v\left(f\left(\mu, \lambda_{1} / \mu, \widetilde{\lambda}\right)-g(\lambda)\right)=v\left(\eta\left(\mu, \lambda_{1} / \mu, \widetilde{\lambda}\right)\right) \notin \Gamma_{\langle\lambda\rangle} .
$$

This means that the infinitesimal $\nu$ is active over $\lambda$, concluding the proof of the theorem.

Proposition 4.7. Let $\lambda=\left(\lambda_{1}, \ldots, \lambda_{m}\right)$ be a regular sequence of infinitesimals with main part $\lambda_{1}, \ldots, \lambda_{k}$, and $\mu$ be an infinitesimal whose valuation does not lie in $\Gamma_{\langle\lambda\rangle}$. Then $\operatorname{dim} \Gamma_{\langle\mu, \lambda\rangle}=k+1$, and thus $\mu, \lambda_{1}, \ldots, \lambda_{m}$ is a regular sequence of infinitesimals with main part $\mu, \lambda_{1}, \ldots, \lambda_{k}$.

The proof is by induction with respect to the difference $l:=m-k$. The assertion is trivial for $l=0$.

For the induction step, consider infinitesimals $\mu$ and $\lambda=\left(\lambda_{1}, \ldots, \lambda_{m}\right)$ such that $\operatorname{dim} \Gamma_{\langle\lambda\rangle}=k-1$ and $\mu, \lambda_{1}, \ldots, \lambda_{k-1}$ form a regular sequence; the difference in question is thus $m-k+1$. Since for the infinitesimals $\mu$ and $\lambda=\left(\lambda_{1}, \ldots, \lambda_{m-1}\right)$ which also satisfy the assumptions of Proposition 4.7, the difference in question is

$$
(m-1)-(k-1)=m-k=l,
$$

we get $\operatorname{dim} \Gamma_{\left\langle\mu, \lambda_{1}, \ldots, \lambda_{m-1}\right\rangle}=k$ by induction hypothesis. We must show that $\operatorname{dim} \Gamma_{\left\langle\mu, \lambda_{1}, \ldots, \lambda_{m}\right\rangle}=k$.

Were $\operatorname{dim} \Gamma_{\left\langle\mu, \lambda_{1}, \ldots, \lambda_{m}\right\rangle}>k$, the infinitesimal $\lambda_{m}$ would be active over the preceding infinitesimals, i.e.

$$
v\left(\lambda_{m}-t\left(\mu, \lambda_{1}, \ldots, \lambda_{m-1}\right)\right) \notin \Gamma_{\left\langle\mu, \lambda_{1}, \ldots, \lambda_{m-1}\right\rangle}=\Gamma_{\left\langle\mu, \lambda_{1}, \ldots, \lambda_{k-1}\right\rangle}
$$

for an $\mathcal{L}$-term $t$. Since $\lambda_{m}$ is non-active over $\lambda_{1}, \ldots, \lambda_{m-1}$, we have

$$
t\left(\mu, \lambda_{1}, \ldots, \lambda_{m-1}\right) \notin\left\langle\lambda_{1}, \ldots, \lambda_{m-1}\right\rangle .
$$

Otherwise we would get a contradiction

$$
v\left(\lambda_{m}-t\left(\mu, \lambda_{1}, \ldots, \lambda_{m-1}\right)\right) \in \Gamma_{\left\langle\lambda_{1}, \ldots, \lambda_{m}\right\rangle}=\Gamma_{\left\langle\lambda_{1}, \ldots, \lambda_{k-1}\right\rangle} .
$$


Further, the induction hypothesis means that the infinitesimals

$$
\mu, \lambda_{1}, \ldots, \lambda_{k-1}, \lambda_{k}, \ldots, \lambda_{m-1}
$$

satisfy the assumptions of the theorem on an active infinitesimal. This implies that the infinitesimal $\nu:=t\left(\mu, \lambda_{1}, \ldots, \lambda_{m-1}\right)$ would be active over $\lambda_{1}, \ldots, \lambda_{m-1}$, and thus

$$
v\left(t\left(\mu, \lambda_{1}, \ldots, \lambda_{m-1}\right)-\tau\left(\lambda_{1}, \ldots, \lambda_{m-1}\right)\right) \notin \Gamma_{\left\langle\lambda_{1}, \ldots, \lambda_{m-1}\right\rangle}=\Gamma_{\left\langle\lambda_{1}, \ldots, \lambda_{k-1}\right\rangle}
$$

for an $\mathcal{L}$-term $\tau$. Then

$$
\begin{aligned}
& v\left(\lambda_{m}-t\left(\mu, \lambda_{1}, \ldots, \lambda_{m-1}\right)\right) \\
& \quad=v\left(\left(\lambda_{m}-\tau\left(\lambda_{1}, \ldots, \lambda_{m-1}\right)\right)-\left(t\left(\mu, \lambda_{1}, \ldots, \lambda_{m-1}\right)-\tau\left(\lambda_{1}, \ldots, \lambda_{m-1}\right)\right)\right) \\
& \quad=\min \left\{v\left(\lambda_{m}-\tau\left(\lambda_{1}, \ldots, \lambda_{m-1}\right)\right), v\left(t\left(\mu, \lambda_{1}, \ldots, \lambda_{m-1}\right)-\tau\left(\lambda_{1}, \ldots, \lambda_{m-1}\right)\right)\right\} .
\end{aligned}
$$

Again, since $\lambda_{m}$ is non-active over $\lambda_{1}, \ldots, \lambda_{m-1}$, we have

$$
v\left(\lambda_{m}-\tau\left(\lambda_{1}, \ldots, \lambda_{m-1}\right)\right) \in \Gamma_{\left\langle\lambda_{1}, \ldots, \lambda_{m-1}\right\rangle}=\Gamma_{\left\langle\lambda_{1}, \ldots, \lambda_{k-1}\right\rangle},
$$

whence both the valuations in the above minimum are distinct and belong to $\Gamma_{\left\langle\mu, \lambda_{1} \ldots, \lambda_{k-1}\right\rangle}$. Consequently,

$$
v\left(\lambda_{m}-t\left(\mu, \lambda_{1}, \ldots, \lambda_{m-1}\right)\right) \in \Gamma_{\left\langle\mu, \lambda_{1}, \ldots, \lambda_{m-1}\right\rangle}=\Gamma_{\left\langle\mu, \lambda_{1}, \ldots, \lambda_{k-1}\right\rangle} .
$$

This contradiction completes the proof of Proposition 4.7.

As an immediate consequence, we obtain:

COROLlary 4.8 (Valuation property for $\mathcal{L}$-terms). If $\lambda=\left(\lambda_{1}, \ldots, \lambda_{m}\right)$ and $\mu$ are infinitesimals, we have the following dichotomy: either

- $\mu$ is non-active over $\lambda$, and then $\Gamma_{\langle\lambda, \mu\rangle}=\Gamma_{\langle\lambda\rangle}$; or

- $\mu$ is active over $\lambda$, and then $\operatorname{dim} \Gamma_{\langle\lambda, \mu\rangle}=\operatorname{dim} \Gamma_{\langle\lambda\rangle}+1$.

In the latter case, one can find an $\mathcal{L}$-term $t(x)$ such that

$$
v(\mu-t(\lambda)) \notin \Gamma_{\langle\lambda\rangle} \quad \text { and } \quad \Gamma_{\langle\lambda, \mu\rangle}=\Gamma_{\langle\lambda\rangle} \oplus \mathbb{Q} \cdot v(\mu-t(\lambda)) .
$$

COROLlary 4.9 (Steinitz's exchange property). Consider a finite number of infinitesimals $\lambda=\left(\lambda_{1}, \ldots, \lambda_{m}\right), \mu, \nu$. If $\nu \in\langle\lambda, \mu\rangle$ and $\nu \notin\langle\lambda\rangle$, then $\mu \in\langle\lambda, \nu\rangle$.

The case where $\mu$ is non-active over the infinitesimals $\lambda$ has been treated as the exchange property for a non-active infinitesimal in Proposition 4.7 and Corollary 3.6. Consider now the other case.

We may, of course, assume that $\lambda_{1}, \ldots, \lambda_{m}$ is a regular sequence of infinitesimals with main part $\lambda_{1}, \ldots, \lambda_{k}$ and that $\mu \notin \Gamma_{\langle\lambda\rangle}$. Then, due to Proposition 4.7 , the infinitesimals $\mu, \lambda_{1}, \ldots, \lambda_{m}$ form a regular sequence with main part $\mu, \lambda_{1}, \ldots, \lambda_{k}$. It follows from the theorem on an active infinitesimal that $\nu$ is active over the infinitesimals $\lambda$. Hence and by the valuation property for 
$\mathcal{L}$-terms (Corollary 4.8), we get

$$
\operatorname{dim} \Gamma_{\langle\lambda, \mu\rangle}=\operatorname{dim} \Gamma_{\langle\lambda, \nu\rangle}=k+1,
$$

and thus the infinitesimal $\mu$ is non-active over the infinitesimals $\lambda, \nu$.

Consider now the equation $\nu-t(\lambda, \mu)=0$. Since $\mu$ is non-active over the infinitesimals $(\lambda, \nu)$, the exchange property for a non-active infinitesimal (cf. Proposition 4.7 and Corollary 3.6) may be applied. Therefore this equation can be solved by means of the implicit function theorem with respect to $\mu$, i.e. $\mu=\tau(\lambda, \nu) \in\langle\lambda, \nu\rangle$ for an $\mathcal{L}$-term $\tau(x, y)$, which is the desired conclusion.

5. Description of Q-subanalytic sets by $\mathcal{L}$-terms. We begin by drawing some conclusions from Steinitz's exchange property (Corollary 4.9). The span operation $s(A):=\langle A\rangle$, which assigns to each subset $A \subset \mathcal{R}$ the substructure generated by $A$, satisfies the following conditions:

(S1) $A \subset B \Rightarrow s(A) \subset s(B)$;

(S2) $b \in s(A) \Rightarrow b \in s\left(a_{1}, \ldots, a_{m}\right)$ for some $a_{1}, \ldots, a_{m} \in A$;

(S3) $A \subset s(A)$;

(S4) $s(s(A))=s(A)$;

(S5) $c \in s(A, b)$ and $c \notin s(A) \Rightarrow b \in s(A, c)$.

Model-theorists call such a span operation $s$ a pregeometry on the structure $\mathcal{R}$ (see e.g. [22]). Conditions (S1)-(S4) are satisfied by algebraic closure in any structure. A first-order structure is called geometric if algebraic closure has the exchange property (S5). Definable closure and algebraic closure coincide in a structure with linear ordering, because in any finite set one can define the least element, the next least element and so on.

Every o-minimal structure satisfies condition (S5) too, and thus is geometric. One can build - by analogy with the dimension of vector spaces or with the transcendence degree of field extensions - a general dimension theory for geometric structures. There is a general notion of independence in such structures. We say that a subset $A$ in $\mathcal{R}$ is free (or independent) if

$$
a \notin s(A \backslash\{a\}) \quad \text { for any } a \in A ;
$$

$A$ is called a basis of $\mathcal{R}$ if it is both a generating system of $\mathcal{R}$ and a free set (cf. [41, Vol. I, Chap. II, $\S 12]$ ). It can be checked that every maximal free subset of an algebraically closed structure $\mathcal{R}$ is a basis, and that any two bases have the same size, called the rank of $\mathcal{R}$. We also have at our disposal the notion of relative rank for a pair of geometric structures $\mathcal{R} \subset \mathcal{S}$.

In our case, the span operation consists just in generating the substructure for subsets of a model $\mathcal{R}$ of the universal theory $T$. Clearly, any ana- 
lytically independent set of infinitesimals in $\mathcal{R}$ is a free set. Therefore the assertion below is a special case of the one for free sets.

Proposition 5.1 (Inversion of analytically independent infinitesimals). Consider two analytically independent sets

$$
\lambda=\left(\lambda_{1}, \ldots, \lambda_{m}\right) \quad \text { and } \quad \mu=\left(\mu_{1}, \ldots, \mu_{m}\right)
$$

of infinitesimals and $m \mathcal{L}$-terms $t(x)=\left(t_{1}(x), \ldots, t_{m}(x)\right), x=\left(x_{1}, \ldots, x_{m}\right)$. If $\mu=t(\lambda)$, then there are $m \mathcal{L}$-terms $\tau_{1}(y), \ldots, \tau_{m}(y), y=\left(y_{1}, \ldots, y_{m}\right)$, such that $\lambda=\tau(\mu)$. In other words, $\langle\lambda\rangle=\langle\mu\rangle$.

As a direct consequence, we obtain

Corollary 5.2. Let $\lambda=\left(\lambda_{1}, \ldots, \lambda_{m}\right)$ be an analytically independent set of infinitesimals and $\mu$ an infinitesimal. Then the set $\left(\lambda_{1}, \ldots, \lambda_{m}, \mu\right)$ is analytically independent iff $\mu \notin\langle\lambda\rangle$.

COROLlary 5.3. A set $\lambda=\left(\lambda_{1}, \ldots, \lambda_{m}\right)$ of infinitesimals is analytically independent iff it is a free set.

This can be easily checked by induction with respect to the number $m$ of infinitesimals.

We now state a theorem concerning the inversion of general special modifications, which asserts that the requirement for the inverse mapping $\psi$ of a special modification $\varphi$ we impose in Section 2 is no constraint on special cubes.

Proposition 5.4. Let $\varphi:(0,1)^{d} \rightarrow S \subset \mathbb{R}^{m}$ be a general special modification, i.e. $\varphi$ is a diffeomorphism of $(0,1)^{d}$ onto $S$ which extends to a $Q$-mapping in the vicinity of $[0,1]^{d}$. If $S$ is described by $\mathcal{L}$-terms, then the inverse mapping $\varphi^{-1}: S \rightarrow(0,1)^{d}$ is piecewise given by $\mathcal{L}$-terms.

For the proof, we shall show that there exists a family $\left(t_{\iota}(y)\right)_{\iota \in I}$ of $\mathcal{L}$ terms, $t_{\iota}(y)=\left(t_{\iota, 1}(y), \ldots, t_{\iota, d}(y)\right)$, such that the infinite disjunction

$$
\bigvee_{\iota \in I}\left[\left(b=\varphi(a) \wedge a \in(0,1)^{d}\right) \Rightarrow\left(a=t_{\iota}(b) \wedge b \in S\right)\right]
$$

holds for any tuples $a \in \mathcal{R}^{d}$ and $b \in \mathcal{R}^{m}$ in an arbitrary model $\mathcal{R}$ of the theory $T$. Then, through model-theoretic compactness, one can find a finite set $\iota_{1}, \ldots, \iota_{n} \in I$ of indices for which the finite disjunction

$$
\bigvee_{k=1, \ldots, n}\left[\left(b=\varphi(a) \wedge a \in(0,1)^{d}\right) \Rightarrow\left(a=t_{\iota_{k}}(b) \wedge b \in S\right)\right]
$$

holds for any such tuples $a$ and $b$ in an arbitrary model $\mathcal{R}$ of the theory $T$. Hence

$$
\left(b=\varphi(a) \wedge a \in(0,1)^{d}\right) \Rightarrow\left[\left(a=t_{\iota_{1}}(b) \vee \cdots \vee a=t_{\iota_{n}}(b)\right) \wedge b \in S\right],
$$


and thus the inverse mapping $\varphi^{-1}$ is piecewise given by $\mathcal{L}$-terms, which is the desired conclusion.

So take any elements $a \in(0,1)^{d}$ and $b \in S^{\mathcal{R}}$ for which $b=\varphi(a)$. We may, of course, confine our analysis to the case where $a=\lambda$ and $b=\mu$ are infinitesimals. Observe that $\operatorname{rk}\langle\lambda\rangle \leq \mathrm{rk}\langle\mu\rangle$, for otherwise $\mu \in T^{\mathcal{R}}$ for a special cube $T$ of dimension $<\operatorname{rk}\langle\lambda\rangle$, whence $\lambda \in\left(\varphi^{-1}(T)\right)^{\mathcal{R}}$ and $\operatorname{dim} \varphi^{-1}(T)=\operatorname{dim} T<\operatorname{rk}\langle\lambda\rangle$, which is impossible.

Consequently, we have

$$
\langle\mu\rangle \subset\langle\lambda\rangle \text { and } \operatorname{rk}\langle\lambda\rangle \leq \operatorname{rk}\langle\mu\rangle,
$$

and thus $\langle\mu\rangle=\langle\lambda\rangle$. Therefore our auxiliary assertion follows and the proof is complete.

Before turning to quantifier elimination for the theory $T$, we state the quasianalytic version of Gabrielov's theorem [13] on the closure and frontier of a semianalytic set, which will be needed in the proof.

TheOrem 5.5 (Gabrielov's closure theorem). If $E \subset \mathbb{R}^{m}$ is a Q-semianalytic set, so are the closure $\bar{E}$ and the frontier $\partial E$. Moreover, if $E$ is of the form

$$
E=\left\{x \in[-1,1]^{m}: f_{1}(x)=\cdots=f_{k}(x)=0, g_{1}(x)>0, \ldots, g_{l}(x)>0\right\},
$$

where $f_{i}$ 's and $g_{j}$ 's are $Q$-analytic functions in the vicinity of the cube $[-1,1]^{m}$, then $\bar{E}$ and $\partial E$ are described by Q-analytic functions which are polynomials in the variables $x$, in the functions $f_{i}$ 's, $g_{j}$ 's and in their (finitely many) partial derivatives.

REMARK 5.6. Gabrielov's proof used a method of truncating Taylor series, which allows one to reduce the problem to sets described by polynomials where the Tarski-Seidenberg theorem applies. This method does not involve the Weierstrass preparation theorem, but relies on the Łojasiewicz inequality instead. Consequently, it can be transferred almost verbatim to the quasianalytic settings.

COROLlary 5.7. If $E \subset \mathbb{R}^{m}$ is a set described by $\mathcal{L}$-terms, so are the closure $\bar{E}$ and the frontier $\partial E$.

It is sufficient, of course, to consider the case of closure. The proof consists then in adding new variables, one for each occurrence of a function symbol involved in a given $\mathcal{L}$-term (as explained in Remark 2.4).

We can now turn to quantifier elimination for the theory $T$.

Theorem 5.8 (on quantifier elimination). Let $\pi: \mathbb{R}_{x}^{m} \times \mathbb{R}_{y}^{n} \rightarrow \mathbb{R}_{x}^{m}$ be the canonical projection. If a set $E \subset \mathbb{R}_{x}^{m} \times \mathbb{R}_{y}^{n}$ is defined by a quantifier-free $\mathcal{L}$-formula $\phi(x, y)$ (i.e. $E$ is described by a finite number of $\mathcal{L}$-terms involved 
in $\phi)$, so is its projection

$$
F=\pi(E)=\left\{x \in \mathbb{R}^{m}: \exists y_{n} \ldots \exists y_{1} \phi(x, y)\right\} .
$$

Accordingly, the theory $T$ admits quantifier elimination.

The proof is by induction with respect to the $\operatorname{dimension} \operatorname{dim} E=: d$. It suffices, of course, to consider the case $n=1$. The case $\operatorname{dim} E=0$ is trivial; take $d \geq 1$. Assuming the assertion to hold for $0,1, \ldots, d-1$, we shall prove it for $d$.

For this purpose, we shall show that there exist a family of quantifier-free formulae $\left(\phi_{\iota}(x)\right)_{\iota \in I}$ such that

$$
\bigwedge_{\iota \in I} \mathcal{R} \models \forall x\left[\phi_{\iota}(x) \Rightarrow \exists y \phi(x, y)\right]
$$

and the infinite disjunction

$$
\bigvee_{\iota \in I}\left[(\exists y \phi(a, y)) \Rightarrow \phi_{\iota}(a)\right]
$$

holds for any fixed tuple $a \in \mathcal{R}^{m}$ in an arbitrary model $\mathcal{R}$ of the theory $T$. Then, through model-theoretic compactness, we get

$$
F=\left\{x \in \mathbb{R}^{m}: \phi_{\iota_{1}}(x)\right\} \cup \cdots \cup\left\{x \in \mathbb{R}^{m}: \phi_{\iota_{l}}(x)\right\}
$$

for some $\iota_{1}, \ldots, \iota_{l} \in I$, which is the desired result.

Obviously, we may assume that the set $E$ is bounded. Take any element

$$
(a, b)=\left(a_{1}, \ldots, a_{m}, b\right) \in E^{\mathcal{R}} \in \mathcal{R}^{m} .
$$

We can, of course, confine our analysis to the case where $(a, b)=(\lambda, \mu)=$ $\left(\lambda_{1}, \ldots, \lambda_{m}, \mu\right)$ are infinitesimals. Note that in non-standard models $\mathcal{R}$ we shall work only with the interpretations $E^{\mathcal{R}}$ of the set $E$, because the set $F$ is not a priori described by $\mathcal{L}$-terms, and thus we are not able to analyse its interpretations yet.

We have two possibilities: either $\mu \in\langle\lambda\rangle$ or $\mu \notin\langle\lambda\rangle$. The former is easy; it yields $\mu=t(\lambda)$ for an $\mathcal{L}$-term $t$, and thus one should attach to our family of formulae one that describes the set $\left(x_{1}, \ldots, x_{m}, t(x)\right)^{-1}(E)$.

The latter needs a more careful treatment. We can reduce our problem to the case where the infinitesimals $\lambda$ are analytically independent. Indeed, one can find a basis chosen from among $\lambda^{\prime}$ s, say $\lambda_{1}, \ldots, \lambda_{r}$ with $r:=\operatorname{rk}\langle\lambda\rangle$. Clearly,

$$
\lambda_{j}=\tau_{j}\left(\lambda_{1}, \ldots, \lambda_{r}\right), \quad j=r+1, \ldots, m,
$$

for some $\mathcal{L}$-terms $\tau_{r+1}, \ldots, \tau_{m}$. Let $\rho: \mathbb{R}^{m} \rightarrow \mathbb{R}^{r}$ be the canonical projection onto the first $r$ coordinates. Then our analysis of the projection $\pi$ of the set $E$ can be replaced by that of the projection $\rho \circ \pi$ of the set

$$
\widetilde{E}:=E \cap\left\{x_{r+1}=\tau_{r+1}\left(x_{1}, \ldots, x_{r}\right)\right\} \cap \cdots \cap\left\{x_{m}=\tau_{m}\left(x_{1}, \ldots, x_{r}\right)\right\},
$$


which is equivalent to that of the projection onto the first $r$ coordinates of the following set also described by $\mathcal{L}$-terms:

$$
\left(x_{1}, \ldots, x_{r}, y\right)(\widetilde{E}) \subset \mathbb{R}^{r} \times \mathbb{R}_{y} .
$$

Hence the reduction goes. The replacement described above is a non-standard counterpart of fibre cutting in the classical subanalytic geometry.

So suppose the infinitesimals $\lambda$ are analytically independent. According to Corollary 5.2, the infinitesimals $(\lambda, \mu) \in E^{\mathcal{R}}$ are analytically independent too, and thus we can assume that $E$ is a special cube of dimension $d=$ $m+1$. Further, due to Gabrielov's closure theorem, the frontier $V:=\partial E$ is described by $\mathcal{L}$-terms of dimension $<d$.

Observe that we can replace the set $E$ by its part lying over the complement of any closed subset $Z \subset \mathbb{R}^{m}$ described by $\mathcal{L}$-terms of dimension $<m$, because the infinitesimals $\lambda$ are analytically independent. Therefore it is sufficient to investigate the parts of the sets $E$ and $V$ over such a complement. Consequently, we can assume that the set $V$ over such a complement is a finite union of special cubes $S_{1}, \ldots, S_{l}$ such that each projection

$$
\pi: S_{i} \rightarrow \mathbb{R}^{m}, \quad i=1, \ldots, l,
$$

is a local Q-diffeomorphism. Indeed, if $V$ is a finite union of special cubes, we should remove the special cubes whose projections onto $\mathbb{R}^{m}$ are of dimension $<m$ and cut out from the remaining special cubes the sets of ramification points, which are described by $\mathcal{L}$-terms and of dimension $<m$.

Further, we can replace the special cubes $S_{i}$ by the sets

$$
S_{i} \backslash \pi^{-1}\left(\pi\left(\partial S_{i}\right)\right), \quad i=1, \ldots, l .
$$

These sets are also described by $\mathcal{L}$-terms due to Gabrielov's closure theorem and the induction hypothesis. Each projection

$$
\pi: S_{i} \backslash \pi^{-1}\left(\pi\left(\partial S_{i}\right)\right) \rightarrow \pi\left(S_{i}\right) \backslash \pi\left(\partial S_{i}\right)
$$

is thus a proper mapping, whence a topological covering.

After decomposition of the sets $\pi\left(S_{i}\right) \backslash \pi\left(\partial S_{i}\right)$ into special cubes and removing those of dimension $<m$, the part of the set $V$ under study is now a finite union of topological covering spaces $V_{C}$ over simply connected open special cubes $C \subset \mathbb{R}^{m}$.

Clearly, each set $V_{C}$ is a finite union of leaves $\Lambda_{1}, \ldots, \Lambda_{n}$ that are the graphs of certain smooth functions $\xi_{1}(x)<\cdots<\xi_{n}(x)$. Then the set

$$
\begin{aligned}
\Lambda & :=\left\{\left(x, y_{1}, \ldots, y_{n}\right):\left(x, y_{1}\right) \in \Lambda_{1}, \ldots,\left(x, y_{n}\right) \in \Lambda_{n}\right\} \\
& =\left\{\left(x, y_{1}, \ldots, y_{n}\right): x \in C,\left(x, y_{1}\right), \ldots,\left(x, y_{n}\right) \in V, y_{1}<\cdots<y_{n}\right\}
\end{aligned}
$$

(which is an open subset of the fibre product of the leaves $\Lambda_{1}, \ldots, \Lambda_{n}$ over $C$ ) is described by $\mathcal{L}$-terms of dimension $m$, and so are its projections $\Lambda_{1}, \ldots, \Lambda_{n}$, again by the induction hypothesis. Observe now that, due to Proposition 5.4 
and the induction hypothesis, the functions $\xi_{1}(x), \ldots, \xi_{n}(x)$ are also piecewise given by $\mathcal{L}$-terms.

We need to consider above only special cubes $C$ such that $\lambda \in C^{\mathcal{R}}$, say $C_{1}, \ldots, C_{s}$. The part of the set $V$ lying over $D:=C_{1} \cap \cdots \cap C_{s}$ is then a finite union of the graphs of certain smooth functions $\xi_{1}(x), \ldots, \xi_{N}(x)$ piecewise given by $\mathcal{L}$-terms. Again, we can cut out the set $W$ of points of $D$ at which any two distinct functions above are equal, because this set is of dimension $<m$. Take the connected component $U$ of $D \backslash W$ (which is described by $\mathcal{L}$-terms through decomposition into special cubes) such that $\lambda \in U^{\mathcal{R}}$. It is obvious that the functions $\xi_{1}(x), \ldots, \xi_{N}(x)$ are totally ordered over $U$, say $\xi_{1}(x)<\cdots<\xi_{N}(x)$ for all $x \in U$.

But then the part $E_{U}$ of the set $E$ lying over $U$ is a finite union of strata between some of the leaves $\Lambda_{k}$ and $\Lambda_{k+1}, k \in K \subset\{1, \ldots, N-1\}$ :

$$
E_{U}=\left\{(x, y): x \in U, \xi_{k}(x)<y<\xi_{k+1}(x) \text { for some } k \in K\right\} .
$$

This can be expressed by means of the following universal $\mathcal{L}$-formula:

$$
(x, y) \in E_{U} \Leftrightarrow\left[x \in U, \bigvee_{k \in K} \xi_{k}(x)<y<\xi_{k+1}(x)\right],
$$

which is true in every model $\mathcal{R}$ of the theory $T$. Therefore $(\lambda, \mu)$ lies in one of those strata, and hence

$$
\xi(\lambda)<\mu<\zeta(\lambda) \text { and }\{\lambda\} \times(\xi(\lambda), \zeta(\lambda)) \subset E^{\mathcal{R}},
$$

for some functions $\xi(x), \zeta(x)$ piecewise given by $\mathcal{L}$-terms. In order to complete the proof, we should attach to our family of formulae one that describes the set

$$
\left(x_{1}, \ldots, x_{m}, t(x)\right)^{-1}(E)
$$

with $t(x):=(\xi(x)+\zeta(x)) / 2$.

Hence and by decomposition into special cubes (see Section 2 and [28]), we obtain immediately

COROllary 5.9. The theory $T$ is complete and o-minimal, and the standard model $\mathcal{R}_{Q}$ is its prime model.

6. Applications. The fact that a universal theory $T$ admits quantifier elimination has weighty model-theoretic and geometric consequences. Every definable function is then piecewise given by terms (a well-known result due to Herbrand [14]; see e.g. [16]):

Proposition 6.1. Consider a first-order language $\mathcal{L}$, a universal theory $T$ in $\mathcal{L}$ and a quantifier-free $\mathcal{L}$-formula $\phi\left(x_{1}, \ldots, x_{m}, y\right)$. If

$$
T \models \forall x_{1} \ldots \forall x_{m} \exists y \phi\left(x_{1}, \ldots, x_{m}, y\right),
$$


then there exist a finite number of terms $t_{1}(x), \ldots, t_{k}(x)$ such that

$$
T \models \forall x_{1} \ldots \forall x_{m} \phi\left(x_{1}, \ldots, x_{m}, t_{1}(x)\right) \vee \cdots \vee \phi\left(x_{1}, \ldots, x_{m}, t_{k}(x)\right) .
$$

COROLlaRY 6.2. If a universal theory $T$ admits quantifier elimination, then for each definable function $f\left(x_{1}, \ldots, x_{m}\right)$ there are finitely many $\mathcal{L}$ terms $t_{1}(x), \ldots, t_{k}(x)$ such that in every model $\mathfrak{M}$ of $T$ we have

$$
\mathfrak{M}=\forall x_{1} \ldots \forall x_{m} f\left(x_{1}, \ldots, x_{m}\right)=t_{1}(x) \vee \cdots \vee f\left(x_{1}, \ldots, x_{m}\right)=t_{k}(x),
$$

i.e. $f$ is piecewise given by the terms $t_{1}(x), \ldots, t_{k}(x)$.

Consequently, the structure $\mathcal{R}_{Q}$ admits smooth cell decomposition piecewise given by $\mathcal{L}$-terms (cf. [28]). Further, the operation of definable closure for subsets of a given model $\mathcal{R}$ of the theory $T$ coincides with that of span discussed in Section 5. We can thus deduce from the valuation property for $\mathcal{L}$-terms (Corollary 4.8) the following general version (cf. $[11,12,26])$ :

TheOREm 6.3 (Valuation property for definable functions). Consider a simple (with respect to definable closure) extension $\mathcal{R} \subset \mathcal{R}\langle a\rangle$ of substructures in a fixed model of the theory $T$. Then we have the following dichotomy:

$$
\text { either } \quad \operatorname{dim} \Gamma_{\mathcal{R}\langle a\rangle}=\operatorname{dim} \Gamma_{\mathcal{R}} \quad \text { or } \quad \operatorname{dim} \Gamma_{\mathcal{R}\langle a\rangle}=\operatorname{dim} \Gamma_{\mathcal{R}}+1 .
$$

In the latter case, one can find an element $r \in \mathcal{R}$ such that

$$
v(a-r) \notin \Gamma_{\mathcal{R}} \quad \text { and } \quad \Gamma_{\mathcal{R}\langle a\rangle}=\Gamma_{\mathcal{R}} \oplus \mathbb{Q} \cdot v(a-r) .
$$

The significance of the valuation property lies to a great extent in its geometric content (see e.g. $[12,26]$ ), namely it is equivalent to the preparation theorem in the sense of Parusiński-Lion-Rolin [32, 19, 33], which says that every definable function of several variables depends piecewise on (or can be prepared with respect to) any fixed variable in a certain simple fashion. The preparation theorem, in turn, yields many geometric, differential and integral applications, like the Lipschitz structure of subanalytic sets (cf. [32, 39]), the log-analytic nature of the volumes of subanalytic sets or asymptotic expansions related to integration (cf. [20, 33, 18]).

The preparation theorem can be derived from the valuation property through model-theoretic compactness and definable choice (cf. $[12,26])$. Note that definable choice is available once we know the theory $T$ is o-minimal. We recall below a version of this theorem for our o-minimal theory $T$ with exponent field $\mathbb{Q}$.

TheOREM 6.4 (Preparation theorem). Consider a definable function $f$ : $\mathcal{R}^{m+1} \rightarrow \mathcal{R}$ and an $\epsilon \in \mathbb{Q}, \epsilon>0$. Then there exist finitely many rational numbers $q_{1}, \ldots, q_{k} \in \mathbb{Q}$ and definable functions

$$
\theta_{1}, \ldots, \theta_{k}, c_{1}, \ldots, c_{k}: \mathcal{R}^{m} \rightarrow \mathcal{R}, \quad u_{1}, \ldots, u_{k}: \mathcal{R}^{m+1} \rightarrow(1-\epsilon, 1+\epsilon) \subset \mathcal{R}
$$


such that for each $x \in \mathcal{R}^{m}$ and $y \in \mathcal{R}$ we have

$$
f(x, y)=\left|y-\theta_{i}(x)\right|^{q_{i}} \cdot c_{i}(x) \cdot u_{i}(x, y) \quad \text { for an } i=1, \ldots, k \text {. }
$$

Another consequence of the description of definable functions by $\mathcal{L}$-terms is a quasi-subanalytic version of Puiseux's theorem with parameter (see [34] for a classical version).

Theorem 6.5 (Puiseux's theorem with parameter). Let $E \subset \mathbb{R}_{x}^{m}$ be a definable subset and $f: E \times(0,1) \rightarrow \mathbb{R}$ be a definable function. Then one can find a cell decomposition of $E$ into finitely many $Q$-cells $C_{1}, \ldots, C_{s}$ (i.e. Q-analytic cells; cf. [28]) for which either

- the function $f_{x}(t):=f(x, t)$ vanishes near zero for all $x \in C_{i}$, or

- there exist $k \in \mathbb{N}, p \in \mathbb{Q}$ and a definable function $F(x, t), Q$-analytic in a neighbourhood $U_{i}$ of $C_{i} \times\{0\} \subset C \times \mathbb{R}_{t}$, such that

(*) $f(x, t)=t^{p} \cdot F\left(x, t^{1 / k}\right) \quad$ and $\quad F(x, 0) \neq 0 \quad$ for all $(x, t) \in U_{i}, x \in C_{i}$.

We may, of course, assume that the function $f(x, t)$ is given by an $\mathcal{L}$-term $\tau(x, t)$. Then the proof is by induction with respect to the complexity of the term $\tau(x, t)$. The theorem is evident if $\tau(x, t)$ is a function symbol of $\mathcal{L}$. So assume that the term $\tau(x, t)$ is composed:

$$
\tau(x, t)=\varphi\left(\tau_{1}(x, t), \ldots, \tau_{r}(x, t)\right),
$$

and that the terms $\tau_{j}(x, t), j=1, \ldots, r$, satisfy condition $(*)$. The case where $\varphi$ is the multiplication function, reciprocal function or root function is easy. What remains is to check the assertion when $\varphi$ is the addition function or a restricted Q-function. We shall only demonstrate how to check the assertion for a restricted Q-function of two variables, because the remaining cases are similar.

Suppose two definable functions $g(x, t)$ and $h(x, t)$ satisfy condition $(*)$. After refining Q-cell decompositions, we can assume that condition $(*)$ is satisfied in a common Q-cell decomposition for both $f$ and $g$, so that

$$
g(x, t)=t^{p} \cdot G\left(x, t^{1 / k}\right), \quad h(x, t)=t^{q} \cdot H\left(x, t^{1 / l}\right) \quad \text { for all }(x, t) \in U_{i},
$$

with obvious assumptions about the numbers $p, q, k, l$ and the functions $G, H$.

Since the problem is non-trivial only when $p, q \geq 0$, we are reduced to the case where

$$
\tau(x, t)=\varphi\left(G\left(x, t^{1 / k}\right), H\left(x, t^{1 / k}\right)\right)
$$

and $G(x, 0), H(x, 0) \in[-1,1]$ for all $x \in C_{i}$. Put

$$
K(x, t):=\varphi(G(x, t), H(x, t))
$$


and

$$
V_{j}:=\left\{x \in C_{i}: 0=K(x, 0)=\frac{\partial K}{\partial t}(x, 0)=\cdots=\frac{\partial^{j-1} K}{\partial t^{j-1}}(x, 0)\right\} .
$$

Then the decreasing sequence of Q-analytic sets $\left(V_{j}\right)_{j \in \mathbb{N}}$ stabilizes (see e.g. $[4,27,28])$ :

$$
C_{i}=V_{0} \supseteq V_{1} \supseteq \cdots \supseteq V_{n}=V_{n+1}=\cdots .
$$

For each $j$, the Q-functions $K_{x}(t):=K(x, t)$ are of constant rank $j$ for all $x \in V_{j} \backslash V_{j+1}$. Therefore, again after partitioning the Q-cell $C_{i}$ into finer Q-cells, we can assume that, for each finer new Q-cell $C$, we have

$$
K(x, t)=t^{j} \cdot F(x, t) \quad \text { for all }(x, t) \in U,
$$

where $U:=U_{i} \cap(C \times \mathbb{R})$ is a neighbourhood of $C \times\{0\} \subset C \times \mathbb{R}_{t}$ and $F(x, t)$ is a definable Q-function in $U$ with $F(x, 0) \neq 0$ for all $x \in C$. Hence

$$
\varphi\left(G\left(x, t^{1 / k}\right), H\left(x, t^{1 / k}\right)\right)=t^{j / k} \cdot F\left(x, t^{1 / k}\right),
$$

which is the desired conclusion.

As a corollary, we immediately obtain

THEOREM 6.6 (Piecewise uniform asymptotics). Under the assumptions of Theorem 6.5 , there exist finitely many rational numbers $q_{1}, \ldots, q_{s} \in \mathbb{Q}$ such that for each $x \in E$, either

- the function $f_{x}(t):=f(x, t)$ vanishes near zero, or

- $f_{x}(t)$ is asymptotic to $c^{q_{i}}$ for some $i=1, \ldots, s$ and $c \in \mathbb{R}, c \neq 0$, i.e.

$$
\lim _{t \rightarrow 0^{+}} \frac{f(x, t)}{c t^{q_{i}}}=1 .
$$

We conclude this article with the following comment. The fundamental method applied in our paper is transformation to normal crossings by blowing up. This method, developed in Zariski's school of algebraic geometry as one of the most powerful tools for the resolution of singularities, culminated in the famous work of Hironaka [15]. The very concept of a normal crossing had originated from ideas of the Italian school of algebraic geometry (see e.g. [40, Chap. I] and [5]).

Acknowledgements. The author wishes to express his gratitude to Wiesław Pawłucki for many discussions concerning this article. We also thank Guillaume Valette for pointing out a gap in our proof of Theorem 4.4 on an active infinitesimal in the previous version of the paper.

This paper was partially supported by Research Project No. N N201 372336 from the Polish Ministry of Science and Higher Education. 


\section{References}

[1] S. S. Abhyankar, On the ramification of algebraic functions, Amer. J. Math. 77 (1955), 575-592.

[2] E. Bierstone and P. D. Milman, Semianalytic and subanalytic sets, Inst. Hautes Études Sci. Publ. Math. 67 (1988), 5-42.

[3] - - - Canonical desingularization in characteristic zero by blowing up the maximum strata of a local invariant, Invent. Math. 128 (1997), 207-302.

[4] - - - Resolution of singularities in Denjoy-Carleman classes, Selecta Math. (N.S.) 10 (2004), 1-28.

[5] O. Chisini, La risoluzione delle singolarità di una superficie mediante trasformazioni birazionali dello spazio, Mem. Accad. Sci. Bologna 8 (1921), 1-42.

[6] H. G. Dales and A. M. Davie, Quasianalytic Banach function algebras, J. Funct. Anal. 13 (1973), 28-50.

[7] Z. Denkowska, S. Łojasiewicz et J. Stasica, Certaines propriétés élémentaires des ensembles sous-analytiques, Bull. Acad. Polon. Sci. Sér. Sci. Math. 27 (1979), 529536.

[8] - - - - Sur le théorème du complémentaire pour les ensembles sous-analytiques, ibid. 27 (1979), 537-539.

[9] J. Denef and L. van den Dries, p-adic and real subanalytic sets, Ann. of Math. 128 (1988), 79-138.

[10] L. van den Dries, A. Macintyre and D. Marker, The elementary theory of restricted analytic fields with exponentiation, ibid. 140 (1994), 183-205.

[11] L. van den Dries and P. Speissegger, The field of reals with multisummable series and the exponential function, Proc. London Math. Soc. (3) 81 (2000), 513-565.

[12] - - - o-minimal preparation theorems, in: Model Theory and its Applications (Ravello, 2002), Quad. Mat. 11, Aracne, Roma, 2002, 87-116.

[13] A. Gabrielov, Complements of subanalytic sets and existential formulas for analytic functions, Invent. Math. 125 (1996), 1-12.

[14] J. Herbrand, Recherches sur la théorie de la démonstration, Trav. Soc. Sci. Lett. Varsovie Cl. III 33 (1930), 1-128.

[15] H. Hironaka, Resolution of singularities of an algebraic variety over a field of characteristic zero, Ann. of Math. 79 (1964), I: 109-203; II: 205-326.

[16] W. Hodges, Model Theory, Cambridge Univ. Press, Cambridge, 1997.

[17] H. W. E. Jung, Darstellung der Funktionen eines algebraischen Körpers zweier unabhängigen Veränderlichen $x, y$ in der Umgebung einer Stelle $x=a, y=b$, J. Reine Angew. Math. 133 (1908), 289-314.

[18] K. Kurdyka et G. Raby, Densité des ensembles sous-analytiques, Ann. Inst. Fourier (Grenoble) 39 (1989), 753-771.

[19] J.-M. Lion et J.-P. Rolin, Théorème de préparation pour les fonctions logarithmicoexponentielles, ibid. 47 (1997), 859-884.

[20] - - - Intégration des fonctions sous-analytiques et volumes des sous-analytiques, ibid. 48 (1999), 755-767.

[21] S. Łojasiewicz, Ensembles semi-analytiques, Inst. Hautes Études Sci., Bures-surYvette, 1964.

[22] D. Macpherson, Notes on o-minimality and variations, in: Model Theory, Algebra and Geometry, Math. Sci. Res. Inst. Publ. 39, Cambridge, 2000, 97-130.

[23] K. J. Nowak, On a universal axiomatization of the real closed fields, Ann. Polon. Math. 65 (1996), 95-103. 
[24] K. J. Nowak, A model-theoretic version of the complement theorem, Bull. Polish Acad. Sci. Math. 47 (1999), 345-354.

[25] -, A model-theoretic version of the complement theorem: applications, ibid. 47 (1999), 355-361.

[26] - A proof of the valuation property and preparation theorem, Ann. Polon. Math. 92 (2007), 75-85.

[27] —, On the Euler characteristic of the links of a set determined by smooth definable functions, ibid. 93 (2008), 231-246.

[28] —, Decomposition into special cubes and its application to quasi-subanalytic geometry, ibid. 96 (2009), 65-74.

[29] -, On two problems concerning quasianalytic Denjoy-Carleman classes, RAAG Preprint 241, 2007, and IMUJ Preprint 19, 2007.

[30] A. G. O'Farrell, Polynomial approximation of smooth functions, J. London Math. Soc. (2) 28 (1983), 496-506.

[31] A. Parusiński, Subanalytic functions, Trans. Amer. Math. Soc. 344 (1994), 583-595.

[32] - , Lipschitz stratification of subanalytic sets, Ann. Sci. École Norm. Sup. 27 (1994), 661-696.

[33] - On the preparation theorem for subanalytic functions, in: New Developments in Singularity Theory (Cambridge, 2000), NATO Sci. Ser. II Math. Phys. Chem. 21, Kluwer, Dordrecht, 2001, 193-215.

[34] W. Pawłucki, Le théorème de Puiseux pour une application sous-analytique, Bull. Polish Acad. Sci. Math. 32 (1984), 555-560.

[35] A. Rambaud, Quasi-analyticité, o-minimalité et élimination des quantificateurs, PhD Thesis, Univ. Paris 7, Paris, 2005; C. R. Math. Acad. Sci. Paris 343 (2006), $1-4$.

[36] J.-P. Rolin, P. Speissegger and A. J. Wilkie, Quasianalytic Denjoy-Carleman classes and o-minimality, J. Amer. Math. Soc. 16 (2003), 751-777.

[37] H. J. Sussmann, Real-analytic desingularization and subanalytic sets: an elementary approach, Trans. Amer. Math. Soc. 317 (1990), 417-461.

[38] V. Thilliez, On quasianalytic local rings, Expo. Math. 26 (2008), 1-23.

[39] G. Valette, Lipschitz triangulations, Illinois J. Math. 49 (2005), 953-979.

[40] O. Zariski, Algebraic Surfaces, 2nd ed., Ergeb. Math. Grenzgeb. 61, Springer, Heidelberg, 1971.

[41] O. Zariski and P. Samuel, Commutative Algebra, Vols. I and II, Van Nostrand, Princeton, 1958, 1960.

Institute of Mathematics

Jagiellonian University

Łojasiewicza 6

30-348 Kraków, Poland

E-mail: nowak@im.uj.edu.pl

Received 7.11.2008

and in final form 10.12.2008 